\title{
Differential Calculus on Compact Matrix Pseudogroups (Quantum Groups)
}

\author{
S. L. Woronowicz \\ Department of Mathematical Methods in Physics, Faculty of Physics, University of Warsaw, \\ Hoża 74, PL-00-682 Warszawa, Poland
}

\begin{abstract}
The paper deals with non-commutative differential geometry. The general theory of differential calculus on quantum groups is developed. Bicovariant bimodules as objects analogous to tensor bundles over Lie groups are studied. Tensor algebra and external algebra constructions are described. It is shown that any bicovariant first order differential calculus admits a natural lifting to the external algebra, so the external derivative of higher order differential forms is well defined and obeys the usual properties. The proper form of the Cartan Maurer formula is found. The vector space dual to the space of left-invariant differential forms is endowed with a bilinear operation playing the role of the Lie bracket (commutator). Generalized antisymmetry relation and Jacobi identity are proved.
\end{abstract}

\section{Introduction}

It is difficult to overestimate the importance of the methods of differential geometry in the theory of Lie groups. Such fundamental notions as tangent Lie algebra and infinitesimal representation are provided by these methods. Infinitesimal representations in turn play an important role in applications of Lie groups in physics: Infinitesimal generators are related to the most important observables such as energy-momentum, angular momentum, and internal quantum numbers.

On the other hand, for differential geometry the Lie group theory is much more than just one of the fields of application. It is sufficient to dip into any contemporary handbook of differential geometry to see that Lie groups lay behind such basic notions as principal and associated fibre bundles. In fact, the development of differential geometry in the past 30 years can be considered as a far reaching implementation of the Erlangen program of F. Klein.

We believe that a similar interplay between differential geometry and group theory will be very fruitful also in the theory of non-commutative spaces. In this case instead of Lie groups one has to consider more general objects: non- 
commutative spaces provided with a group structure, i.e. quantum groups [3] (pseudogroups in the sense of [7] and [10]). Moreover, following the general ideas of Connes [2] one should use the notion of differential form (rather than that of vector field) as the basic one.

The main aim of this paper is the presentation of differential calculus of differential forms on a quantum group. We show that all important notions and formulae of classical Lie group theory admit a generalization to the quantum group case. To be more specific we restrict ourselves to compact matrix pseudogroups as introduced in [10], although we believe that the compactness is not crucial to the problems considered here.

We use the axiomatic method to introduce the first order differential calculus. The axioms contain the basic properties of external derivative (Leibnitz rule) as well as additional requirements related to the underlying group structure (left-and right-covariance). The axiomatics is not categorical: for a given quantum group there exist many non-equivalent differential calculi. This fact is in unpleasant contrast with the more satisfactory situation in classical differential geometry where the notions of first order differential form and external derivative are introduced in a constructive (functorial) way. At the moment after many unsuccessful attempts we accord with the opinion that for the non-commutative geometry no such functorial definition is possible. We have, however, to stress that once the first order differential calculus is fixed, all further notions are introduced in a fully functorial way.

We briefly describe the content of the paper. Section 1 contains the main definitions and results concerning first order differential calculus. We indicate that reasonable calculi are in one to one correspondence with right ideals $\mathscr{R}$ in the algebra of "smooth functions" on considered quantum group satisfying certain conditions. The problem of the right choice of $\mathscr{R}$ will be considered in a forthcoming paper. Here we notice only that if the ideal $\mathscr{R}$ is too small then the corresponding differential calculus is over multidimensional, i.e. very complicated from the computational point of view. If $\mathscr{R}$ is too large then the resulting differential calculus may become trivial. In this case the infinitesimal generators may not contain enough information to restore the original representation.

Section 2 is of a more general character. The notion of bicovariant bimodule investigated in this section corresponds on the classical level to that of vector fibre bundle over the group manifold endowed with the left and right action of the group. It is well known that any such bundle is topologically trivial: There exists a family of left-invariant sections such that for any point of the group the values of the sections at this point form a basis in the fibre over this point. We shall prove the corresponding result in the general case (see Propositions 2.1 and 2.3). Moreover, considering the right shifts of left-invariant sections we arrive at the notion of adjoint representation. The results of this section provide the proper tools for the proofs of the theorems of Sect. 1 as well as for further studies related to tensor and external algebras.

The main role in Sect. 3 is played by the twisted flip automorphism $\sigma: \Gamma \otimes \Gamma \rightarrow \Gamma \otimes \Gamma$ associated with any bicovariant bimodule $\Gamma$. We prove that $\sigma$ satisfies the braid equation. Due to this equation we shall be able to introduce the antisymmetrization operation acting on tensor powers of $\Gamma$, and consequently to 
carry out the external algebra construction. In Sect. 4 we prove that any bicovariant first order differential calculus admits a natural lifting to higher order differential forms.

Section 5 is devoted to the vector space $T$ dual to the space of left-invariant differential forms. It plays the role of tangent Lie algebra. Using the automorphism $\sigma$ considered in Sect. 3 we shall introduce the "commutator" of any two elements of $T$. It satisfies the twisted antisymmetry relation and twisted Jacobi identity. Cartan Maurer formulae are also derived. The results of Sect. 5 may be used to introduce the notion of enveloping algebra. We would like to stress that in our theory the relations defining the enveloping algebra remain quadratic [cf. (5.25)] like in the classical case. On the other hand, the approach to quantum group theory presented in [3] and [4] uses the quantized enveloping algebra introduced by relations involving entire analytic functions. It would be interesting to relate these different points of view. Some results in this direction are obtained by Rosso [6] and Koornwinder [5].

This paper contains only a few examples illustrating the general theory. All the examples concern the twisted $S U(2)$ group, and even in this particular case the detailed computations are not explicitly presented. This gap will be filled by the forthcoming papers where the differential calculus on the twisted $S U(N)$ group will be investigated. It turns out that the general theory presented in this paper can be used to produce non-standard differential calculi on classical Lie groups.

To end this Introduction we would like to formulate a duality principle that in many cases enables us to omit boring proofs.

For any $g \in M_{N}(\mathbf{C})$ we denote by $g^{t}$ the transpose of $g$. If $G \subset M_{N}(\mathbf{C})$ is a group of matrices, then $G^{\prime}=\left\{g^{t}: g \in G\right\}$ is also a group of matrices. A similar fact holds for compact matrix pseudogroups. Let $G=(A, u)$ be a compact matrix pseudogroup and $u^{t}$ be the transpose of $u:\left(u^{t}\right)_{k l}=u_{l k}(k, l=1,2, \ldots, N)$. Then $G^{\prime}=\left(A, u^{t}\right)$ is a compact matrix pseudogroup [one can check that the comultiplication $\Phi^{\prime}$ and coinverse $\kappa^{\prime}$ associated with $G^{\prime}$ are given by the formulae $\Phi^{\prime}=\sigma_{A} \circ \Phi$ and $\kappa^{\prime}=\kappa^{-1}$, where $\Phi$ and $\kappa$ are the comultiplication and coinverse associated with $G$ and $\sigma_{A}$ is the flip automorphism of $A \otimes A: \sigma_{A}(a \otimes b)=b \otimes a$ for any $\left.a, b \in A\right]$. This fact leads to the following LR-duality principle (LR stands for Left-Right).

If $D E F$ is a definition of a notion of the theory of compact matrix pseudogroups, then replacing in DEF all notions related to a pseudogroup $G$ by the corresponding notions related to $G^{\prime}$ we obtain a definition of a new notion related to $G$. We say that this new notion is the LR-dual of the old one. For example $\sigma_{A} \circ \Phi$ and $\kappa^{-1}$ are LR-duals of $\Phi$ and $\kappa$, respectively. Similarly, if THM is a theorem of the theory of compact matrix pseudogroups, then replacing in THM all notions by their LR-duals we obtain a new theorem of the theory. For example in Sect. 1, Proposition 1.3 is the LR-dual of Proposition 1.2.

Throughout the paper we deal with many pairs of LR-dual definitions and statements. We believe that the reader will easily correctly identify these pairs.

\section{First Order Differential Calculus}

The first order differential forms on a smooth manifold $M$ are sections of the cotangent bundle $T^{*}(M)$. The set of all sections $\Gamma(M)=C^{\infty}\left(T^{*}(M)\right)$ is a bimodule 
over $C^{\infty}(M)$. The external derivative

$$
d: C^{\infty}(M) \rightarrow \Gamma(M)
$$

obeys the basic property: $d(a b)=(d a) b+a d b$ for any $a, b \in C^{\infty}(M)$. Moreover (at least for compact manifolds), any differential form $\varrho \in \Gamma(M)$ is of the form $\varrho=\sum a_{k} d b_{k}$, where $a_{k}, b_{k} \in C^{\infty}(M)(k=1,2, \ldots, K)$. Expressing this situation in more abstract terms we arrive at the following

Definition 1.1. Let $\mathscr{A}$ be an algebra with unity, $\Gamma$ be a bimodule over $\mathscr{A}$ and

$$
d: \mathscr{A} \rightarrow \Gamma
$$

be a linear map. We say that $(\Gamma, d)$ is a first order differential calculus over $\mathscr{A}$ if 1. For any $a, b \in \mathscr{A}$,

$$
d(a b)=(d a) b+a d b .
$$

2. Any element $\varrho \in \Gamma$ is of the form

$$
\varrho=\sum_{k=1}^{K} a_{k} d b_{k}
$$

where $a_{k}, b_{k} \in \mathscr{A} ; k=1,2, \ldots, K ; K$ is a natural number.

Two first order differential calculi $(\Gamma, d)$ and $\left(\Gamma^{\prime}, d^{\prime}\right)$ over $\mathscr{A}$ are said to be identical if there exists a bimodule isomorphism $i: \Gamma \rightarrow \Gamma^{\prime}$ such that $i(d a)=d^{\prime} a$ for any $a \in \mathscr{A}$. In what follows we shall identify identical calculi.

Let $\mathscr{A}$ be an algebra, $m: \mathscr{A} \otimes \mathscr{A} \rightarrow \mathscr{A}$ be the multiplication map [i.e. linear mapping such that $m(a \otimes b)=a b$ for any $a, b \in \mathscr{A}]$ and

$$
\mathscr{A}^{2}=\{q \in \mathscr{A} \otimes \mathscr{A}: m q=0\} .
$$

Clearly $\mathscr{A}^{2}$ is a linear subset of $\mathscr{A} \otimes \mathscr{A}$. Setting (for any $c \in \mathscr{A}$ )

$$
\begin{gathered}
c\left(\sum_{k=1}^{K} a_{k} \otimes b_{k}\right)=\sum_{k=1}^{K} c a_{k} \otimes b_{k}, \\
\left(\sum_{k=1}^{K} a_{k} \otimes b_{k}\right) c=\sum_{k=1}^{K} a_{k} \otimes b_{k} c,
\end{gathered}
$$

we introduce on $\mathscr{A}^{2}$ an $\mathscr{A}$-bimodule structure. For any $b \in \mathscr{A}$ we put $D b=I \otimes b-b \otimes I$. One can easily check that $D: \mathscr{A} \rightarrow \mathscr{A}^{2}$ is a linear mapping and that $\left(\mathscr{A}^{2}, D\right)$ is a first order differential calculus over $\mathscr{A}$. From the practical point of view this calculus is not very interesting. Its theoretical importance is revealed by the following simple.

Proposition 1.1. Let $\mathscr{N}$ be a sub-bimodule of $\mathscr{A}^{2}, \Gamma=\mathscr{A}^{2} / \mathcal{N}, \pi$ be the canonical epimorphism $\mathscr{A}^{2} \rightarrow \Gamma$ and $d=\pi \circ D$. Then $(\Gamma, d)$ is a first order differential calculus over $\mathscr{A}$. Any first order differential calculus over $\mathscr{A}$ can be obtained in this way.

Proof. Only the last remark needs a proof. Let $(\Gamma, d)$ be a first order differential calculus over $\mathscr{A}$. For any $\sum_{k} a_{k} \otimes b_{k} \in \mathscr{A}^{2}$ we have

$$
\begin{gathered}
\sum_{k} c a_{k} d b_{k}=c \sum_{k} a_{k} d b_{k} \\
\sum_{k} a_{k} d\left(b_{k} c\right)=\left(\sum_{k} a_{k} d b_{k}\right) c+\left(\sum_{k} a_{k} b_{k}\right) d c=\left(\sum_{k} a_{k} d b_{k}\right) c
\end{gathered}
$$


These relations show that the mapping $\pi: \mathscr{A}^{2} \rightarrow \Gamma$ induced by the formula

$$
\pi\left(\sum_{k} a_{k} \otimes b_{k}\right)=\sum_{k} a_{k} d b_{k}
$$

is a bimodule homomorphism. We shall show that $\pi$ is surjective. Indeed, if $\varrho \in \Gamma$, then $\varrho=\sum a_{k} d b_{k}$ (where $a_{k}, b_{k} \in \mathscr{A} ; \sum$ denotes the sum over $k=1,2, \ldots, K$ ). Clearly, $\sum a_{k} \otimes b_{k}-\left(\sum a_{k} b_{k}\right) \otimes I \in \mathscr{A}^{2}$ and $\pi\left(\sum a_{k} \otimes b_{k}-\left(\sum a_{k} b_{k}\right) \otimes I\right)=\varrho-\left(\sum a_{k} b_{k}\right) d I=\varrho[$ the relation $d I=0$ follows immediately from (1.2)]. Let

$$
\mathscr{N}=\left\{\sum_{k} a_{k} \otimes b_{k} \in \mathscr{A}^{2}: \sum_{k} a_{k} d b_{k}=0\right\}
$$

be the kernel of $\pi$. Then $\Gamma$ may be identified with $\mathscr{A}^{2} / \mathscr{N}$ and for any $b \in \mathscr{A}$ we have [cf. (1.6)]

$$
\pi(D b)=\pi(I \otimes b-b \otimes I)=I d b-b d I=d b . \quad \text { Q.E.D. }
$$

The main subject of this paper is the investigation of differential calculus on compact matrix pseudogroups (quantum groups). Let $G=(A, u)$ be such a group. We shall use the notation introduced in [10]. In particular, $\Phi$ is the comultiplication and $\kappa$ is the coinverse associated with $G$ and $\mathscr{A}$ is the $*$-algebra generated by matrix elements of $u$. Moreover, $e$ will denote the $*$-character defined $\mathscr{A}$ such that $e\left(u_{k l}\right)=\delta_{k l}(k, l=1,2, \ldots, N)$. We shall treat $\mathscr{A}$ as the set of all $C^{\infty}$ functions defined on $G$. This means that $\mathscr{A}$ defines the smooth structure on $G$. By definition differential calculus on $G$ is a differential calculus over $\mathscr{A}$. The group structure existing on $G$ implies some natural conditions that should be satisfied by any reasonable differential calculus on $G$. The covariance conditions introduced below are the most important.

If $\Gamma$ is an $\mathscr{A}$-bimodule, then $\mathscr{A} \otimes \Gamma$ (and $\Gamma \otimes \mathscr{A})$ carries a natural $(\mathscr{A} \otimes \mathscr{A})$ bimodule structure. For example $(a \otimes b)(c \otimes \varrho)=a c \otimes b \varrho$ for any $a, b, c \in \mathscr{A}$ and $\varrho \in \Gamma$.

Definition 1.2. Let $(\Gamma, d)$ be a first order differential calculus on $G$. We say that $(\Gamma, d)$ is left-covariant if

$$
\left(\sum a_{k} d b_{k}=0\right) \Rightarrow\left(\sum \Phi\left(a_{k}\right)(\mathrm{id} \otimes d) \Phi\left(b_{k}\right)=0\right)
$$

for any $a_{k}, b_{k} \in \mathscr{A}(k=1,2, \ldots, K)$.

The content of this definition is revealed by the following

Proposition 1.2. Let $(\Gamma, d)$ be a left-covariant first order differential calculus on $G$. Then there exists a linear mapping

$$
\Phi_{\Gamma}: \Gamma \rightarrow \mathscr{A} \otimes \Gamma
$$

such that

1. For any $a \in \mathscr{A}$ and $\varrho \in \Gamma$,

$$
\begin{aligned}
& \Phi_{\Gamma}(a \varrho)=\Phi(a) \Phi_{\Gamma}(\varrho), \\
& \Phi_{\Gamma}(\varrho a)=\Phi_{\Gamma}(\varrho) \Phi(a) .
\end{aligned}
$$


2. The diagram

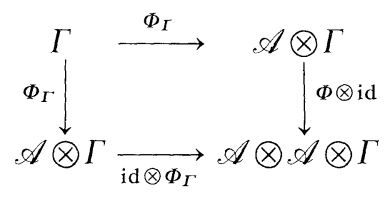

is commutative.

3. For any $\varrho \in \Gamma$,

$$
(e \otimes \mathrm{id}) \Phi_{\Gamma}(\varrho)=\varrho
$$

4. The diagram

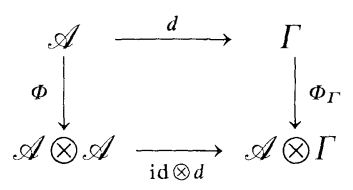

is commutative.

The linear mapping (1.9) is determined uniquely by (1.10) and Condition 4.

Proof. Using (1.10) and Condition 4 one can easily check that

$$
\Phi_{\Gamma}\left(\sum a_{k} d b_{k}\right)=\sum \Phi\left(a_{k}\right)(\mathrm{id} \otimes d) \Phi\left(b_{k}\right)
$$

for any $a_{k}, b_{k} \in \mathscr{A}$. This proves the uniqueness of (1.9). On the other hand, the implication (1.8) shows that $\Phi_{\Gamma}$, as introduced by (1.15), is a well defined linear mapping from $\Gamma$ into $\mathscr{A} \otimes \Gamma$. Equation (1.10) and the commutativity of (1.14) follows immediately from (1.15).

Let $a, b \in \mathscr{A}$. Using (1.15) we have

$$
\begin{aligned}
(\Phi \otimes \mathrm{id}) \Phi_{\Gamma}(a d b) & =(\Phi \otimes \mathrm{id})[\Phi(a)(\mathrm{id} \otimes d) \Phi(b)] \\
& =(\Phi \otimes \mathrm{id}) \Phi(a)(\mathrm{id} \otimes \mathrm{id} \otimes d)(\Phi \otimes \mathrm{id}) \Phi(b) .
\end{aligned}
$$

On the other hand, using (1.15) twice we get

$$
\begin{aligned}
\left(\mathrm{id} \otimes \Phi_{\Gamma}\right) \Phi_{\Gamma}(a d b) & =\left(\mathrm{id} \otimes \Phi_{\Gamma}\right)[\Phi(a)(\mathrm{id} \otimes d) \Phi(b)] \\
& =(\mathrm{id} \otimes \Phi) \Phi(a)(\mathrm{id} \otimes \mathrm{id} \otimes d)(\mathrm{id} \otimes \Phi) \Phi(b),
\end{aligned}
$$

and (A.1) of the Appendix shows that the diagram (1.12) is commutative.

Let $a, b \in \mathscr{A}$. Remembering that $e$ is multiplicative and using (A.5) we obtain

$$
\begin{aligned}
(e \otimes \mathrm{id}) \Phi_{\Gamma}(a d b) & =(e \otimes \mathrm{id})[\Phi(a)(\mathrm{id} \otimes d) \Phi(b)] \\
& =[(e \otimes \mathrm{id}) \Phi(a)] d[(e \otimes \mathrm{id}) \Phi(b)]=a d b,
\end{aligned}
$$

and (1.13) follows.

Let $a, b, c \in \mathscr{A}$. Then $(a d b) c=a d(b c)-a b d c$, and using (1.15) we have

$$
\begin{aligned}
\Phi_{\Gamma}((a d b) c) & =\Phi(a)(\mathrm{id} \otimes d) \Phi(b c)-\Phi(a b)(\mathrm{id} \otimes d) \Phi(c) \\
& =\Phi(a)[(\mathrm{id} \otimes d) \Phi(b c)-\Phi(b)(\mathrm{id} \otimes d) \Phi(c)] \\
& =\Phi(a)[(\mathrm{id} \otimes d) \Phi(b)] \Phi(c)=\Phi_{\Gamma}(a d b) \Phi(c),
\end{aligned}
$$

and (1.11) follows. Q.E.D. 
Definition 1.3. Let $(\Gamma, d)$ be a first order differential calculus on $G$. We say that $(\Gamma, d)$ is right-covariant if

$$
\left(\sum a_{k} d b_{k}=0\right) \Rightarrow\left(\sum \Phi\left(a_{k}\right)(d \otimes \mathrm{id}) \Phi\left(b_{k}\right)=0\right)
$$

for any $a_{k}, b_{k} \in \mathscr{A}(k=1,2, \ldots, K) .(\Gamma, d)$ is said to be bicovariant if it is left- and rightcovariant.

Using the same techniques as in the proof of Proposition 1.2 one can prove

Proposition 1.3. Let $(\Gamma, d)$ be a right-covariant first order differential calculus on $G$. Then there exists a linear mapping

$$
\Gamma_{\Gamma} \Phi: \Gamma \rightarrow \Gamma \otimes \mathscr{A}
$$

such that

1. For any $a \in \mathscr{A}$ and $\varrho \in \Gamma$,

$$
\begin{aligned}
& { }_{\Gamma} \Phi(a \varrho)=\Phi(a)_{\Gamma} \Phi(\varrho), \\
& { }_{\Gamma} \Phi(\varrho a)={ }_{\Gamma} \Phi(\varrho) \Phi(a) .
\end{aligned}
$$

2. The diagram

is commutative.

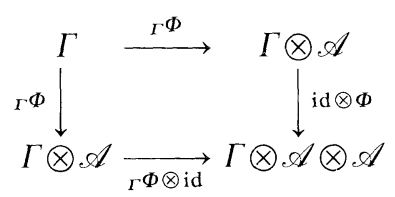

3. For any $\varrho \in \Gamma$,

$$
(\mathrm{id} \otimes e)_{\Gamma} \Phi(\varrho)=\varrho
$$

4. The diagram

is commutative.

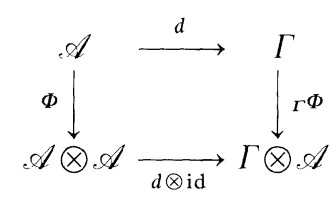

The linear mapping (1.17) is determined uniquely by (1.18) and Condition 4.

Clearly,

$$
{ }_{\Gamma} \Phi\left(\sum a_{k} d b_{k}\right)=\sum \Phi\left(a_{k}\right)(d \otimes \mathrm{id}) \Phi\left(b_{k}\right)
$$

for any $a_{k}, b_{k} \in \mathscr{A}$.

We also have

Proposition 1.4. Let $(\Gamma, d)$ be a bicovariant first order differential calculus on $G$ and $\Phi_{\Gamma}$ and ${ }_{\Gamma} \Phi$ be linear mappings introduced by Proposition 1.2 and Proposition 1.3, respectively. Then the diagram

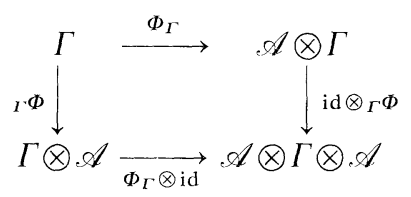

is commutative. 
Proof. Let $a, b \in \mathscr{A}$. Using (1.15) and (1.19) we have

$$
\begin{aligned}
& \left(\mathrm{id} \otimes_{\Gamma} \Phi\right) \Phi_{\Gamma}(a d b)=\left(\mathrm{id} \otimes_{\Gamma} \Phi\right)[\Phi(a)(\mathrm{id} \otimes d) \Phi(b)] \\
& =[(\mathrm{id} \otimes \Phi) \Phi(a)](\mathrm{id} \otimes d \otimes \mathrm{id})[(\mathrm{id} \otimes \Phi) \Phi(b)] .
\end{aligned}
$$

On the other hand, using (1.19) and (1.15) we obtain

$$
\begin{aligned}
\left(\Phi_{\Gamma} \otimes \mathrm{id}\right)_{\Gamma} \Phi(a d b) & =\left(\Phi_{\Gamma} \otimes \mathrm{id}\right)[\Phi(a)(d \otimes \mathrm{id}) \Phi(b)] \\
& =[(\Phi \otimes \mathrm{id}) \Phi(a)](\mathrm{id} \otimes d \otimes \mathrm{id})[(\Phi \otimes \mathrm{id}) \Phi(b)],
\end{aligned}
$$

and (A.1) shows that the diagram (1.20) is commutative. Q.E.D.

The covariance conditions imply some restrictions on the sub-bimodule $\mathscr{N}$ considered in Proposition 1.1. To formulate these restrictions we shall use the linear mappings $r$ and $s$ acting on $\mathscr{A} \otimes \mathscr{A}$ introduced in [10] and the adjoint action of $G$ on itself denoted by ad (cf. [9]). Let us recall that, by definition, for any $a, b \in \mathscr{A}$ :

$$
\begin{aligned}
& r(a \otimes b)=(a \otimes I) \Phi(b), \\
& s(a \otimes b)=(I \otimes a) \Phi(b) .
\end{aligned}
$$

Mappings $r$ and $s$ are bijections, for example the inverse of $r$ is given by $(a, b \in \mathscr{A})$,

$$
r^{-1}(a \otimes b)=(a \otimes I)(\kappa \otimes \mathrm{id}) \Phi(b) .
$$

More useful formulae describing properties of these mappings can be found in the Appendix.

Theorem 1.5. Let $\mathscr{R}$ be a right ideal of $\mathscr{A}$ contained in kere and $\mathscr{N}=r^{-1}(\mathscr{A} \otimes \mathscr{R})$. Then $\mathscr{N}$ is a sub-bimodule of $\mathscr{A}^{2}$. Moreover, let $\Gamma=\mathscr{A}^{2} / \mathscr{N}$, $\pi$ be the canonical epimorphism $\mathscr{A}^{2} \rightarrow \Gamma$ and $d=\pi \circ D$. Then the first order differential calculus $(\Gamma, d)$ is left-covariant. Any left-covariant first order differential calculus on $G$ can be obtained in this way.

The proof will be given in Sect. 2 .

Examples. 1. If $G$ is a Lie group then setting $\mathscr{R}=(\text { ker } e)^{2}$ we obtain the usual differential calculus on $G$.

2. The differential calculus on the twisted $S U(2)$ group presented in [8] is leftcovariant. In this case the right ideal $\mathscr{R}$ is generated by six elements: $\alpha^{*}+\mu^{2} \alpha$ $-\left(1+\mu^{2}\right) I, \gamma^{2}, \gamma^{*} \gamma, \gamma^{* 2},(\alpha-I) \gamma$, and $(\alpha-I) \gamma^{*}$ (cf. Proposition 2.4 in [8]). In the following this calculus will be called $3 D$-calculus on $S_{\mu} U(2)$ [one can check that $\operatorname{dim}(\operatorname{ker} e / \mathscr{R})=3]$.

3. Let $G=S_{\mu} U(2)$ and $\mathscr{R}_{+}\left(\mathscr{R}_{-}\right.$, respectively) be the right ideal in $\mathscr{A}$ generated by the following nine elements:

$$
\begin{gathered}
\gamma^{2}, \gamma\left(\alpha^{*}-\alpha\right), \mu^{2} \alpha^{* 2}-\left(1+\mu^{2}\right)\left(\alpha \alpha^{*}-\gamma^{*} \gamma\right)+\alpha^{2}, \gamma^{*}\left(\alpha^{*}-\alpha\right), \gamma^{* 2}, \\
a \gamma, a\left(\alpha^{*}-\alpha\right), a \gamma^{*}, \\
a\left(\mu^{2} \alpha+\alpha^{*}-\left(1+\mu^{2}\right) I\right),
\end{gathered}
$$


where $a=\mu^{2} \alpha+\alpha^{*}-\left[\left(1+\mu^{4}\right) / \mu\right] I\left(a=\mu^{2} \alpha+\alpha^{*}+\left[\left(1+\mu^{4}\right) / \mu\right] I\right.$, respectively). One can check that $\mathscr{R}_{ \pm} C$ kere and $\operatorname{dim}\left(\operatorname{ker} e / \mathscr{R}_{ \pm}\right)=4$. The corresponding differential calculi on $S_{\mu} U(2)$ will be called $4 D_{ \pm}$-calculi.

Theorem 1.6. Let $\mathscr{R}$ be a right ideal of $\mathscr{A}$ contained in kere and $\mathscr{N}=s^{-1}(\mathscr{R} \otimes \mathscr{A})$. Then $\mathscr{N}$ is a sub-bimodule of $\mathscr{A}^{2}$. Moreover, let $\Gamma=\mathscr{A}^{2} / \mathscr{N}, \pi$ be the canonical epimorphism $\mathscr{A}^{2} \rightarrow \Gamma$, and $d=\pi \circ D$. Then the first order differential calculus $(\Gamma, d)$ is right-covariant. Any right-covariant first order differential calculus on $G$ can be obtained in this way.

The proof will be given in Sect. 2 .

To formulate the next theorem we need the concept of ad-invariance.

Let

$$
\text { ad }: \mathscr{A} \rightarrow \mathscr{A} \otimes \mathscr{A}
$$

be the adjoint action of $G$ onto itself introduced in [9]. We recall that for any $a \in \mathscr{A}$ :

$$
\operatorname{ad}(a)=s\left(r^{-1}(I \otimes a)\right) .
$$

Combining (1.22) and (1.23) one can easily check that

$$
\operatorname{ad}(a)=\sum_{k} b_{k} \otimes \kappa\left(a_{k}\right) c_{k},
$$

where $a_{k}, b_{k}$, and $c_{k}(k=1,2, \ldots, K)$ are elements of $\mathscr{A}$ such that

$$
(\mathrm{id} \otimes \Phi) \Phi(a)=\sum_{k} a_{k} \otimes b_{k} \otimes c_{k} .
$$

Using (1.29) and standard properties of comultiplication and coinverse [cf. (A.1), (A.2)] one can check that the diagram

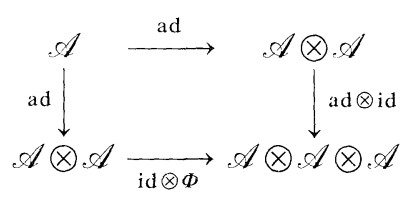

is commutative.

We say that a linear subset $T \subset \mathscr{A}$ is ad-invariant if $\operatorname{ad}(T) \subset T \otimes \mathscr{A}$. We shall use

Lemma 1.7. Let $T$ be a linear ad-invariant subset of $\mathscr{A}$ and $\mathscr{R}$ be a right ideal of $\mathscr{A}$ generated by $T$. Then $\mathscr{R}$ is ad-invariant.

Proof. Let $a, b \in \mathscr{A}$. We claim that

$$
\operatorname{ad}(a b)=\sum_{k}\left(I \otimes \kappa\left(b_{k}^{\prime}\right)\right) \operatorname{ad}(a) \Phi\left(b_{k}^{\prime \prime}\right),
$$

where $b_{k}^{\prime}, b_{k}^{\prime \prime}(k=1,2, \ldots, K)$ are elements of $\mathscr{A}$ such that

$$
\Phi(b)=\sum_{k} b_{k}^{\prime} \otimes b_{k}^{\prime \prime} .
$$

Indeed, using (1.23) and remembering that $\kappa$ is antimultiplicative we see that

$$
r^{-1}(I \otimes a b)=\sum_{k}\left(\kappa\left(b_{k}^{\prime}\right) \otimes I\right) r^{-1}(I \otimes a)\left(I \otimes b_{k}^{\prime \prime}\right) .
$$


Inserting this expression into (1.28) and using (A.13) and (A.14), we obtain (1.31). The assertion of the lemma follows easily from (1.31). Q.E.D.

Theorem 1.8. Let $\mathscr{R}$ be a right ideal of $\mathscr{A}$ contained in kere and $(\Gamma, d)$ be the leftcovariant first order differential calculus described in Theorem 1.5. Then $(\Gamma, d)$ is bicovariant if and only if $\mathscr{R}$ is ad-invariant.

The proof will be given in Sect. 2 .

The standard differential calculus on Lie groups is bicovariant. The $3 D$ calculus on $S_{\mu} U(2)$ described in [8] is not bicovariant. This fact does not incapacitate this calculus; due to the computational simplicity it remains a useful tool in many problems related to twisted $S U(2)$. One has to stress, however, that the general theory presented in this paper is not applicable to $3 D$-calculus, so some of the results obtained in [8] (e.g. the existence of the higher order calculus) remain mysterious.

Let $T_{2}$ ( $T_{1}$ and $T_{0}$, respectively) be the linear subsets of $\mathscr{A}$ spanned by (1.24) [(1.25) and (1.26), respectively]. One can check that $T_{s}(s=0,1,2)$ are ad-invariant. In fact, the adjoint action of $G$ on $T_{s}$ is equivalent to the representation of spin $S$ (note that $\operatorname{dim} T_{s}=2 s+1$ ). Therefore, according to Lemma 1.7 the ideals $\mathscr{R}_{ \pm}$are adinvariant and $4 D_{ \pm}$-calculi are bicovariant. These calculi as well as the corresponding calculi on twisted $S U(N)$ will be investigated in a separate paper.

To end this section we present some definitions and results related to *-structure.

Definition 1.4. Let $(\Gamma, d)$ be a first order differential calculus on $G$. We say that $(\Gamma, d)$ is a $*$-calculus if

$$
\left(\sum a_{k} d b_{k}=0\right) \Rightarrow\left(\sum d\left(b_{k}^{*}\right) a_{k}^{*}=0\right)
$$

for any $a_{k}, b_{k} \in \mathscr{A}(k=1,2, \ldots, K)$.

We have

Proposition 1.9. Let $(\Gamma, d)$ be a first order differential *-calculus on $G$. Then there exists unique antilinear involution

$$
\Gamma \ni \varrho \rightarrow \varrho^{*} \in \Gamma
$$

such that

$$
\begin{aligned}
& (a \varrho)^{*}=\varrho^{*} a^{*}, \\
& (\varrho a)^{*}=a^{*} \varrho^{*}, \\
& (d a)^{*}=d\left(a^{*}\right)
\end{aligned}
$$

for any $\varrho \in \Gamma$ and $a \in \mathscr{A}$. In other words, $\Gamma$ is $a *$-bimodule and $d$ intertwines the $*$-operations on $\mathscr{A}$ and $\Gamma$. Moreover, using the induced $*$-structure on $\mathscr{A} \otimes \Gamma$ and $\Gamma \otimes \mathscr{A}(c f .(2.57)$ and (2.59)) we have

1. If $(\Gamma, d)$ is left-covariant, then using the notation introduced in Proposition 1.2, for any $\varrho \in \Gamma$ we have

$$
\Phi_{\Gamma}\left(\varrho^{*}\right)=\left(\Phi_{\Gamma}(\varrho)\right)^{*} .
$$

In other words, $\left(\Gamma, \Phi_{\Gamma}\right)$ is a left-covariant *-bimodule. 
2. If $(\Gamma, d)$ is right-covariant, then using the notation introduced in Proposition 1.3, for any $\varrho \in \Gamma$, we have

$$
\left.{ }_{\Gamma} \Phi\left(\varrho^{*}\right)={ }_{\Gamma} \Phi(\varrho)\right)^{*} .
$$

In other words, $\left(\Gamma,{ }_{\Gamma} \Phi\right)$ is a right-covariant $*$-bimodule.

Proof. Using (1.34) and (1.36) one can easily check that

$$
\left(\sum a_{k} d b_{k}\right)^{*}=\sum d\left(b_{k}^{*}\right) a_{k}^{*}
$$

for any $a_{k}, b_{k} \in \mathscr{A}$. This proves the uniqueness of (1.33). On the other hand, the implication (1.32) shows that $*$ as introduced by (1.39) is a well defined antilinear mapping from $\Gamma$ into $\Gamma$. Equations (1.34) and (1.36) follow immediately from (1.39), and (1.35) can be checked by direct computation: if $\varrho=b d c$ (where $b, c \in \mathscr{A})$, then

$$
\begin{aligned}
(\varrho a)^{*} & =(b(d c) a)^{*}=(b d(c a)-b c d a)^{*} \\
& =d\left((c a)^{*}\right) b^{*}-d\left(a^{*}\right)(b c)^{*} \\
& =d\left(a^{*} c^{*}\right) b^{*}-d\left(a^{*}\right) c^{*} b^{*}=a^{*} \varrho^{*},
\end{aligned}
$$

and (1.35) follows. Using (1.35) and Definition (1.39) one easily checks that $*$ is an involution. Similarly, one can verify relations (1.37) and (1.38). Q.E.D.

We also have

Theorem 1.10. Let $\mathscr{R}$ be a right ideal of $\mathscr{A}$ contained in kere and $(\Gamma, d)$ be the leftcovariant first order differential calculus described in Theorem 1.5. Then $(\Gamma, d)$ is a *-calculus if and only if $\kappa(x)^{*} \in \mathscr{R}$ for any $x \in \mathscr{R}$.

The proof will be given in Sect. 2 .

Using this criterion one can easily check that all examples of differential calculi considered in this section are $*$-calculi.

\section{Covariant Bimodules}

Let $T$ be a vector bundle over a Lie group $G$. In many cases the left and the right actions of $G$ on itself admit a natural lifting to $T$. This is the case when $T$ is a bundle obtained from the tangent bundle by one of the operations considered in the tensor algebra. Then we have the natural actions (left and right) of $G$ on the set of all smooth sections of $T$. In the pseudogroup case $G=(A, u)$ this situation is described by the following definitions:

Definition 2.1. Let $\Gamma$ be a bimodule over $\mathscr{A}$ and $\Phi_{\Gamma}: \Gamma \rightarrow \mathscr{A} \otimes \Gamma$ be a linear map. We say that $\left(\Gamma, \Phi_{\Gamma}\right)$ is a left-covariant bimodule if

1. For any $a \in \mathscr{A}$ and $\varrho \in \Gamma$,

$$
\begin{aligned}
& \Phi_{\Gamma}(a \varrho)=\Phi(a) \Phi_{\Gamma}(\varrho), \\
& \Phi_{\Gamma}(\varrho a)=\Phi_{\Gamma}(\varrho) \Phi(a) .
\end{aligned}
$$


2. The diagram

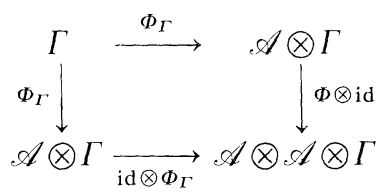

is commutative.

3. For any $\varrho \in \Gamma$,

$$
(e \otimes \mathrm{id}) \Phi_{\Gamma}(\varrho)=\varrho .
$$

Definition 2.2. Let $\Gamma$ be a bimodule over $\mathscr{A}$ and $\Gamma: \Gamma \rightarrow \Gamma \otimes \mathscr{A}$ be a linear map. We say that $\left(\Gamma,{ }_{\Gamma} \Phi\right)$ is a right-covariant bimodule if

1. For any $a \in \mathscr{A}$ and $\varrho \in \Gamma$,

$$
\begin{aligned}
& { }_{\Gamma} \Phi(a \varrho)=\Phi(a)_{\Gamma} \Phi(\varrho), \\
& { }_{\Gamma} \Phi(\varrho a)={ }_{\Gamma} \Phi(\varrho) \Phi(a) .
\end{aligned}
$$

2. The diagram

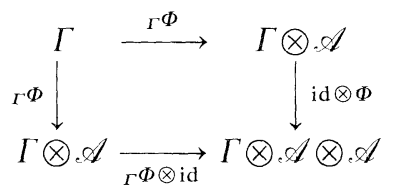

is commutative.

3. For any $\varrho \in \Gamma$,

$$
(\mathrm{id} \otimes e)_{\Gamma} \Phi(\varrho)=\varrho .
$$

Definition 2.3. Let $\Gamma$ be a bimodule over $\mathscr{A}$ and $\Phi_{\Gamma}: \Gamma \rightarrow \mathscr{A} \otimes \Gamma$ and ${ }_{\Gamma} \Phi: \Gamma \rightarrow \Gamma \otimes \mathscr{A}$ be linear maps. We say that $\left(\Gamma, \Phi_{I},{ }_{\Gamma} \Phi\right)$ is a bicovariant bimodule if

1. $\left(\Gamma, \Phi_{\Gamma}\right)$ is a left-covariant bimodule.

2. $\left(\Gamma,{ }_{\Gamma} \Phi\right)$ is a right-covariant bimodule.

3. The diagram

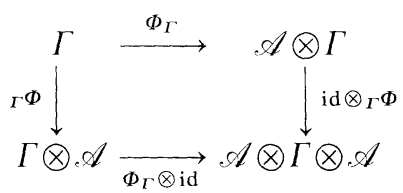

is commutative.

Let $\left(\Gamma, \Phi_{\Gamma}\right)$ be a left-covariant bimodule over $\mathscr{A}$. An element $\omega \in \Gamma$ is said to be left-invariant if

$$
\Phi_{\Gamma}(\omega)=I \otimes \omega .
$$

We denote by ${ }_{\text {inv }} \Gamma$ the set of all left-invariant elements of $\Gamma$. Clearly, ${ }_{\text {inv }} \Gamma$ is a linear subspace of $\Gamma$. We have

Theorem 2.1. Let $\left(\Gamma, \Phi_{\Gamma}\right)$ be a left-covariant bimodule over $\mathscr{A}$ and $\left(\omega_{i}\right)_{i \in I}$ be a basis in the vector space of all left-invariant elements of $\Gamma$. Then 
1. Any element $\varrho \in \Gamma$ is of the form

$$
\underline{Q}=\sum_{i \in I} a_{i} \omega_{i},
$$

where the $a_{i} \in \mathscr{A}(i \in I)$ are uniquely determined.

2. Any element $\varrho \in \Gamma$ is of the form

$$
\varrho=\sum_{i \in I} \omega_{i} b_{i},
$$

where $b_{i} \in \mathscr{A}(i \in I)$ are uniquely determined.

3. There exist linear functionals $f_{i j} \in \mathscr{A}^{\prime}(i, j \in I)$ such that

$$
\begin{gathered}
\omega_{i} b=\sum_{j \in I}\left(f_{i j} * b\right) \omega_{j}, \\
a \omega_{i}=\sum_{j \in I} \omega_{j}\left(\left(f_{i j} \circ \kappa^{-1}\right) * a\right)
\end{gathered}
$$

for any $a, b \in \mathscr{A}$ and $i \in I$. These functionals are determined uniquely by (2.13). They satisfy the following relation:

$$
f_{i j}(a b)=\sum_{h \in I} f_{i k}(a) f_{k j}(b)
$$

for any $i, j \in I$ and $a, b \in \mathscr{A}$. Moreover,

$$
f_{i J}(I)=\delta_{i J}
$$

Remark. We do not assume that $\operatorname{dim}_{\text {inv }} \Gamma<\infty$. In general, $I$ is an infinite set. However, in any case when we perform a summation over $I$, only a finite number of terms do not vanish. In the worst cases, when we sum functionals, sums are pointwise finite.

First we shall prove

Lemma 2.2. Let $\left(\Gamma, \Phi_{\Gamma}\right)$ be a left-covariant bimodule over $\mathscr{A}$ and ${ }_{\mathrm{inv}} \Gamma \subset \Gamma$ be the subspace of all left-invariant elements of $\Gamma$. Then there exists a unique projection

$$
P: \Gamma \rightarrow_{\text {inv }} \Gamma
$$

such that

$$
P(b \varrho)=e(b) P(\varrho)
$$

for any $b \in \mathscr{A}$ and $\varrho \in \Gamma$. Moreover, for any $\varrho \in \Gamma$ we have

$$
\varrho=\sum_{k} a_{k} P\left(\varrho_{k}\right)
$$

where $a_{k}\left(\varrho_{k}\right.$, respectively) are elements of $\mathscr{A}(\Gamma$, respectively) such that

$$
\Phi_{\Gamma}(\varrho)=\sum_{k} a_{k} \otimes \varrho_{k} .
$$

Proof. For any $\varrho \in \Gamma$ we set

$$
P(\varrho)=\sum_{k} \kappa\left(a_{k}\right) \varrho_{k}
$$

where $a_{k} \in \mathscr{A}$ and $\varrho_{k} \in \Gamma(k=1,2, \ldots, K)$ are chosen in such a way that $(2.20)$ holds. Clearly, $P$ is a linear mapping acting on $\Gamma$. We shall prove that $P(\varrho)$ is left-invariant 
for any $\varrho \in \Gamma$. Let

$$
\begin{gathered}
\Phi\left(a_{k}\right)=\sum_{l} a_{k l} \otimes b_{k l}, \\
\Phi_{\Gamma}\left(\varrho_{k}\right)=\sum_{m} c_{k m} \otimes \varrho_{k m},
\end{gathered}
$$

where $a_{k l}, b_{k l}, c_{k m} \in \mathscr{A} ; \varrho_{k m} \in \Gamma$. Commutativity of the diagram (2.3) shows that

$$
\sum_{k, l} a_{k l} \otimes b_{k l} \otimes \varrho_{k}=\sum_{k, m} a_{k} \otimes c_{k m} \otimes \varrho_{k m} .
$$

Moreover, using (A.22) we see that

$$
\sum_{l} \Phi\left(\kappa\left(a_{k l}\right)\right)\left(b_{k l} \otimes I\right)=I \otimes \kappa\left(a_{k}\right) .
$$

Now using (2.1) and the above formulae we compute:

$$
\begin{aligned}
\Phi_{\Gamma}(P(\varrho)) & =\sum_{k} \Phi\left(\kappa\left(a_{k}\right)\right) \Phi_{\Gamma}\left(\varrho_{k}\right)=\sum_{k \cdot m} \Phi\left(\kappa\left(a_{k}\right)\right)\left(c_{k m} \otimes \varrho_{k m}\right) \\
& =\sum_{k \cdot l} \Phi\left(\kappa\left(a_{k l}\right)\right)\left(b_{k l} \otimes \varrho_{k}\right)=I \otimes \sum_{k} \kappa\left(a_{k}\right) \varrho_{k} \\
& =I \otimes P(\varrho) .
\end{aligned}
$$

This shows that $P(\varrho)$ is left-invariant. If $\varrho$ is left-invariant then decomposition $(2.20)$ takes the form $\Phi_{\Gamma}(\varrho)=I \otimes \varrho$ and definition (2.21) shows that (2.17) is a projection.

Now we shall prove (2.18). Let $b \in \mathscr{A}$ and

$$
\Phi(b)=\sum_{n} b_{n}^{\prime} \otimes b_{n}^{\prime \prime},
$$

where $b_{n}^{\prime}, b_{n}^{\prime \prime} \in \mathscr{A}$. Then

$$
\Phi_{I}(b \varrho)=\sum_{n, k} b_{n}^{\prime} a_{k} \otimes b_{n}^{\prime \prime} \varrho_{k}
$$

and

$$
P(b \varrho)=\sum_{n, k} \kappa\left(b_{n}^{\prime} a_{k}\right) b_{n}^{\prime \prime} \varrho_{k} .
$$

Remembering that $\kappa$ is antimultiplicative and using (A.3) we obtain

$$
P(b \varrho)=\sum_{n, k} \kappa\left(a_{k}\right) \kappa\left(b_{n}^{\prime}\right) b_{n}^{\prime \prime} \varrho_{k}=e(b) \sum_{k} \kappa\left(a_{k}\right) \varrho_{k}=e(b) P(\varrho) .
$$

Formula (2.19) can also be checked by direct computation. Taking into account (2.22) we see that

$$
P\left(\varrho_{k}\right)=\sum_{m} \kappa\left(c_{k m}\right) \varrho_{k m}
$$

and using (2.23), (A.4), and (2.4) we obtain

$$
\begin{aligned}
\sum_{k} a_{k} P\left(\varrho_{k}\right) & =\sum_{k, m} a_{k} \kappa\left(c_{k m}\right) \varrho_{k m}=\sum_{k \cdot l} a_{k l} k\left(b_{k l}\right) \varrho_{k} \\
& =\sum_{k} e\left(a_{k}\right) \varrho_{k}=(e \otimes \mathrm{id}) \Phi_{\Gamma}(\varrho)=\varrho .
\end{aligned}
$$

If $P^{\prime}: \Gamma \stackrel{\text { onto }}{\longrightarrow}$ inv $\Gamma$ is another projection such that $P^{\prime}(b \varrho)=e(b) P^{\prime}(\varrho)$, then applying $P^{\prime}$ to the both sides of (2.19) we obtain [cf. (2.4)]

$$
P^{\prime}(\varrho)=\sum_{k} e\left(a_{k}\right) P\left(\varrho_{k}\right)=P\left(\sum_{k} e\left(a_{k}\right) \varrho_{k}\right)=P\left((e \otimes \mathrm{id}) \Phi_{\Gamma}(\varrho)\right)=P(\varrho) .
$$

This shows the uniqueness of (2.17). 
Proof of Theorem 2.1. Ad1. The existence of the decomposition (2.11) follows from (2.19). To prove the uniqueness assume that $\varrho \in \Gamma$ is given by (2.11). Then [cf. (2.1) and (2.10)],

$$
\Phi_{\Gamma}(\varrho)=\sum_{i \in I} \Phi\left(a_{i}\right)\left(I \otimes \omega_{i}\right)
$$

and using (2.18), the relation $P\left(\omega_{i}\right)=\omega_{\imath}$ and (A.6) we obtain

$$
(\mathrm{id} \otimes P) \Phi_{\Gamma}(\varrho)=\sum_{i \in I}(\mathrm{id} \otimes e) \Phi\left(a_{i}\right) \otimes \omega_{i}=\sum_{i \in I} a_{i} \otimes \omega_{i}
$$

Remembering that $\omega_{i}(i \in I)$ are linearly independent we see that the coefficients $a_{i} \in \mathscr{A}$ are uniquely determined. This proves the uniqueness of the decomposition (2.11).

Ad3. For any $b \in \mathscr{A}$ and $j \in I, \omega_{j} b$ admits a decomposition of the form (2.11). Let $F_{j i}(b)$ denote the coefficient preceding $\omega_{i}$ in this decomposition:

$$
\omega_{j} b=\sum_{i \in I} F_{j i}(b) \omega_{i} .
$$

Clearly, the $F_{j i}(i, j \in I)$ are linear mappings acting on $\mathscr{A}$. For any $a, b \in \mathscr{A}$ and any $j \in I$, we have

$$
\sum_{i \in I} F_{j i}(a b) \omega_{i}=\omega_{j} a b=\sum_{h \in I} F_{j h}(a) \omega_{h} b=\sum_{h, i \in I} F_{j h}(a) F_{h i}(b) \omega_{i}
$$

and using the uniqueness of the decomposition (2.11) we obtain

$$
F_{j i}(a b)=\sum_{h \in I} F_{j h}(a) F_{h i}(b)
$$

for all $i, j \in I$ and $a, b \in \mathscr{A}$.

Let $f_{j i}(i, j \in I)$ be the linear functional, defined on $\mathscr{A}$, introduced by the formula

$$
f_{j i}(a)=e\left(F_{j i}(a)\right)
$$

for any $a \in \mathscr{A}$.

Applying $e$ to the both sides of (2.25) we get (2.15). Inserting in (2.24) $b=I$ we see that $F_{j i}(I)=\delta_{j i} I$ and (2.16) follows.

To prove (2.13) we apply $\Phi_{\Gamma}$ to both sides of (2.24). Using (2.2), (2.10), and (2.1) we get

$$
\left(I \otimes \omega_{j}\right) \Phi(b)=\sum_{i \in I} \Phi\left(F_{j i}(b)\right)\left(I \otimes \omega_{i}\right) .
$$

On the other hand, by virtue of (2.24) we have

$$
\left(I \otimes \omega_{j}\right) \Phi(b)=\sum_{i \in I}\left(\mathrm{id} \otimes F_{j i}\right) \Phi(b)\left(I \otimes \omega_{i}\right) .
$$

Comparing the two formulae we see that

$$
\Phi\left(F_{j i}(b)\right)=\left(\mathrm{id} \otimes F_{j i}\right) \Phi(b)
$$

for any $b \in \mathscr{A}$ and $i, j \in I$. Applying $(\mathrm{id} \otimes e)$ to both sides of this equation we obtain [cf. (A.6)],

$$
F_{j i}(b)=\left(\mathrm{id} \otimes f_{i i}\right) \Phi(b)=f_{i i} * b .
$$

Inserting this result into (2.24) we obtain (2.13). 
Let $i, h \in I$. In order to prove (2.14) we have to show that

$$
\sum_{j \in I} f_{j i} *\left(f_{h j} \circ \kappa^{-1}\right)=\delta_{i h} e .
$$

Let $a \in \mathscr{A}$. Then

$$
\begin{aligned}
\sum_{j \in I} f_{j i} *\left(f_{h j} \circ \kappa^{-1}\right)(\kappa(a)) & =\sum_{j \in I}\left(f_{j i} \otimes\left(f_{h j} \circ \kappa^{-1}\right)\right) \Phi(\kappa(a)) \\
& =\sum_{j \in I}\left[f_{j i} \otimes\left(f_{h j} \circ \kappa^{-1}\right)\right] \sigma(\kappa \otimes \kappa) \Phi(a) \\
& =\sum_{j \in I}\left(f_{h j} \otimes f_{j i}\right)(\mathrm{id} \otimes \kappa) \Phi(a) .
\end{aligned}
$$

Formula (2.15) means that $\sum_{j \in I} f_{h j} \otimes f_{j i}=f_{h i} m$. On the other hand [cf. (A.4) and (A.7)], we have $m(\mathrm{id} \otimes \kappa) \Phi(a)=e(\kappa(a)) I$. Therefore [cf. (2.16)], the expression (2.27) equals to $\delta_{h i} e(\kappa(a))$ and (2.26) follows. In the same way one can check that

$$
\sum_{j \in I}\left(f_{j h} \circ \kappa^{-1}\right) * f_{i j}=\delta_{h i} e .
$$

Inserting in (2.13) $b=\left(f_{h_{j}} \circ \kappa^{-1}\right) * a$, summing over $j$ and using (2.26) we obtain (2.14).

Ad2. The existence of the decomposition (2.12) follows immediately from Statement 1 and formula (2.14). To prove the uniqueness assume that for some $b_{i}$ $\left(i \in I\right.$, only a finite number of $b_{i}$ are different from 0$)$ we have

$$
\sum_{i \in I} \omega_{t} b_{i}=0
$$

We have to show that all $b_{i}=0(i \in I)$. Using (2.13) and the uniqueness of (2.11) we obtain

$$
\sum_{i \in I} f_{i j} * b_{i}=0
$$

for all $j \in I$. Computing the convolution product with $f_{j h} \circ \kappa^{-1}$, summing over $j$ and using (2.28) we obtain $b_{h}=0$ for all $h \in I$. Q.E.D.

Theorem 2.1 gives the complete description of left-covariant bimodules. Using (2.13) and (2.1) we have

$$
\begin{gathered}
\left(\sum_{i \in I} a_{i} \omega_{i}\right) b=\sum_{i j \in I} a_{i}\left(f_{i j} * b\right) \omega_{j}, \\
\Phi_{\Gamma}\left(\sum_{i \in I} a_{i} \omega_{i}\right)=\sum_{i \in I} \Phi\left(a_{i}\right)\left(I \otimes \omega_{i}\right) .
\end{gathered}
$$

If $\left(f_{i j}\right)_{i j \in I}$ is a family of functionals defined on $\mathscr{A}$ satisfying relations (2.15) and (2.16), then considering the free left module $\Gamma$ over $\mathscr{A}$ generated by $\omega_{i}(i \in I)$ and using the above formulae to introduce the right multiplication by elements of $\mathscr{A}$ and the left action of $G$ we obtain a left covariant bimodule.

Let $\left(\Gamma,{ }_{\Gamma} \Phi\right)$ be a right-covariant bimodule over $\mathscr{A}$. An element $\eta \in \Gamma$ is said to be right-invariant if

$$
{ }_{I} \Phi(\eta)=\eta \otimes I
$$


We denote by $\Gamma_{\mathrm{inv}}$ the set of all right-invariant elements of $\Gamma$. Clearly, $\Gamma_{\mathrm{inv}}$ is a linear subspace of $\Gamma$. The following theorem gives the complete description of right covariant bimodules.

Theorem 2.3. Let $\left(\Gamma,{ }_{\Gamma} \Phi\right)$ be a right-covariant bimodule over $\mathscr{A}$ and $\left(\eta_{i}\right)_{i \in I}$ be a basis in the vector space of all right-invariant elements of $\Gamma$. Then

1. Any element $\varrho \in \Gamma$ is of the form

$$
\varrho=\sum_{i \in I} a_{i} \eta_{i},
$$

where $a_{i} \in \mathscr{A}(i \in I)$ are uniquely determined.

2. Any element $\varrho \in \Gamma$ is of the form

$$
\varrho=\sum_{i \in I} \eta_{i} b_{i},
$$

where $b_{i} \in \mathscr{A}(i \in I)$ are uniquely determined.

3. There exist linear functionals $f_{i j} \in \mathscr{A}^{\prime}(i, j \in I)$ such that

$$
\begin{gathered}
\eta_{i} b=\sum_{j \in I}\left(b * f_{i j}\right) \eta_{j}, \\
a \eta_{i}=\sum_{j \in I} \eta_{j}\left(a *\left(f_{i j} \circ \kappa^{-1}\right)\right)
\end{gathered}
$$

for any $a, b \in \mathscr{A}$ and $i \in I$. These functionals are determined uniquely by (2.33). They satisfy the following relation:

$$
f_{i j}(a b)=\sum_{k \in I} f_{i k}(a) f_{k j}(b)
$$

for any $i, j \in I$ and $a, b \in \mathscr{A}$. Moreover,

$$
f_{i j}(I)=\delta_{i j} .
$$

Proof. Apply the duality principle (see Sect. 0) to Theorem 2.1 or repeat the proof of Theorem 2.1 with the necessary modifications. In the latter case one proves at first Statement 2, then 3, and at the end Statement 1. Q.E.D.

For bicovariant bimodules besides Theorems 2.1 and 2.3 we have additionally

Theorem 2.4. Let $\left(\Gamma, \Phi_{\Gamma},{ }_{\Gamma} \Phi\right)$ be a bicovariant bimodule over $\mathscr{A}$ and $\left(\omega_{i}\right)_{i \in I}$ be a basis in the vector space of all left-invariant elements of $\Gamma$. Then

1. For any $i \in I$,

$$
{ }_{\Gamma} \Phi\left(\omega_{i}\right)=\sum_{j \in I} \omega_{j} \otimes R_{j i},
$$

where $R_{j i} \in \mathscr{A}(i, j \in I)$ satisfy the following relations

$$
\begin{gathered}
\Phi\left(R_{j i}\right)=\sum_{h \in I} R_{j h} \otimes R_{h i}, \\
e\left(R_{j i}\right)=\delta_{j i} .
\end{gathered}
$$

2. There exists a basis $\left(\eta_{i}\right)_{i \in I}$ (indexed by the same set $I$ ) in the vector space of all right-invariant elements of $\Gamma$ such that

$$
\omega_{i}=\sum_{j \in I} \eta_{j} R_{j i}
$$

for all $i \in I$. 
3. With this choice of basis in $\Gamma_{\mathrm{inv}}$, the functionals $f_{i j}(i, j \in I)$ introduced in Theorems 2.1 and 2.3 coincide.

4. For any $j, h \in I$ and any $a \in \mathscr{A}$,

$$
\sum_{i \in I} R_{i j}\left(a * f_{i h}\right)=\sum_{i \in I}\left(f_{j i} * a\right) R_{h i} .
$$

Proof. Ad1. Using the commutativity of (2.9) we see that

$$
\left(\Phi_{\Gamma} \otimes \mathrm{id}\right)_{\Gamma} \Phi\left(\omega_{i}\right)=\left(\mathrm{id} \otimes_{\Gamma} \Phi\right) \Phi_{\Gamma}\left(\omega_{i}\right)=\left(\mathrm{id} \otimes_{\Gamma} \Phi\right)\left(I \otimes \omega_{i}\right)=I \otimes_{\Gamma} \Phi\left(\omega_{i}\right) .
$$

It shows that ${ }_{\Gamma} \Phi\left(\omega_{i}\right) \in_{\text {inv }} \Gamma \otimes \mathscr{A}$ and formula (2.35) follows. Applying (id $\left.\otimes \Phi\right)$ to the both sides of (2.35) and using (2.7) we obtain

$$
\sum_{j \in I} \omega_{j} \otimes \Phi\left(R_{j i}\right)=\sum_{h \in I} \Gamma \Phi\left(\omega_{h}\right) \otimes R_{h i}=\sum_{j h \in I} \omega_{j} \otimes R_{j h} \otimes R_{h i},
$$

and formula (2.36) follows. To prove (2.37) it is sufficient to apply (id $\otimes e$ ) to the both sides of (2.35) and use (2.8).

Ad2. Inserting in (A.3) and (A.4) $a=R_{i j}(i, j \in I)$ and using (2.36) and (2.37) we obtain

$$
\begin{aligned}
& \sum_{h \in I} \kappa\left(R_{i h}\right) R_{h j}=\delta_{i j} I, \\
& \sum_{h \in I} R_{i h} \kappa\left(R_{h j}\right)=\delta_{i j} I .
\end{aligned}
$$

For any $j \in I$, let

$$
\eta_{j}=\sum_{i \in I} \omega_{i} \kappa\left(R_{i j}\right)
$$

Then formula (2.38) follows immediately from (2.40). Using (2.6), (2.35), (2.36), (A.2), and (2.41) we compute

$$
\begin{aligned}
{ }_{\Gamma} \Phi\left(\eta_{j}\right) & =\sum_{i \in I} \Phi\left(\omega_{i}\right) \Phi\left(\kappa\left(R_{i j}\right)\right)=\sum_{i, h, k}\left(\omega_{h} \otimes R_{h i}\right) \kappa\left(R_{k j}\right) \otimes \kappa\left(R_{i k}\right) \\
& =\sum_{i, h, k} \omega_{h} \kappa\left(R_{k j}\right) \otimes R_{h i} \kappa\left(R_{i k}\right)=\sum_{h, k} \omega_{h} \kappa\left(R_{k j}\right) \otimes \delta_{h k} I=\eta_{j} \otimes I .
\end{aligned}
$$

It means that $\eta_{j}(j \in I)$ are right-invariant.

Let $\eta \in \Gamma$ be right-invariant. According to Theorem 2.1.2 and (2.38),

$$
\eta=\sum_{j \in I} \eta_{j} b_{j}
$$

where $b_{j} \in \mathscr{A}(j \in I)$. If $\eta=0$ then [cf. (2.42) and Theorem 2.1.2] $\sum_{j \in I} \kappa\left(R_{i j}\right) b_{j}=0(i \in I)$ and using (2.41) we get $b_{j}=0$ for all $j \in I$. This shows that the decomposition (2.43) is unique. Applying ${ }_{\Gamma} \Phi$ to the both sides of (2.43) and using (2.31) and (2.6) we get

$$
\eta \otimes I=\sum_{j \in I}\left(\eta_{j} \otimes I\right) \Phi\left(b_{j}\right) .
$$

Comparing this formula with (2.43) we get $\Phi\left(b_{j}\right)=b_{j} \otimes I$ and [cf. (A.5)] $b_{j}=e\left(b_{j}\right) I$ for all $j \in I$. This way we proved that any $\eta \in \Gamma_{\mathrm{inv}}$ is a unique linear combination of $\eta_{j}(j \in I)$. Therefore, $\left(\eta_{j}\right)_{j \in I}$ is a basis in $\Gamma_{\mathrm{inv}}$ and Statement 2 is proven. 
Ad3. In this part of the proof the functionals introduced in Theorem 2.3.3 will be denoted by $\tilde{f}_{j i}$. So Eq. (2.34) takes the form

$$
a \eta_{i}=\sum_{j \in I} \eta_{j}\left(a *\left(\tilde{f}_{i j} \circ \kappa^{-1}\right)\right) .
$$

Using (2.42) and (2.14) we obtain

$$
\sum_{i, h} \omega_{h}\left(\left(f_{i h^{\circ}} \kappa^{-1}\right) * a\right) \kappa\left(R_{i j}\right)=\sum_{i, h} \omega_{h} \kappa\left(R_{h i}\right)\left(a *\left(\tilde{f}_{j i} \circ \kappa^{-1}\right)\right) .
$$

Therefore (cf. Theorem 2.1.2),

$$
\sum_{i \in I}\left[\left(f_{i h} \circ \mathcal{K}^{-1}\right) * a\right] \kappa\left(R_{i j}\right)=\sum_{i \in I} \kappa\left(R_{h i}\right)\left(a *\left(\tilde{f}_{j i} \circ \kappa^{-1}\right)\right) .
$$

We know that $e(f * a)=e(a * f)=f(a)$ for any $a \in \mathscr{A}$ and $f \in \mathscr{A}^{\prime}$ [cf. (A.9)]. Therefore, applying $e$ to both sides of (2.44), remembering that $e$ is multiplicative and using (A.7) and (2.37) we get $f_{j h}\left(\kappa^{-1}(a)\right)=\tilde{f}_{j h}\left(\kappa^{-1}(a)\right)$ for all $a \in \mathscr{A}$. It shows that $f_{j h}=\tilde{f}_{i h}$.

Ad4. Applying $\kappa^{-1}$ to the both sides of (2.44) we get

$$
\sum_{i \in I} R_{i j} \kappa^{-1}\left(\left(f_{i h} \circ \kappa^{-1}\right) * a\right)=\sum_{i \in I} \kappa^{-1}\left(a *\left(f_{i i} \circ \kappa^{-1}\right)\right) R_{h i} .
$$

We compute

$$
\begin{aligned}
\kappa^{-1}\left(\left(f_{i h} \circ \kappa^{-1}\right) * a\right) & =\left(\mathrm{id} \otimes f_{i h}\right)\left(\kappa^{-1} \otimes \kappa^{-1}\right) \Phi(a) \\
& =\left(f_{i h} \otimes \mathrm{id}\right) \Phi\left(\kappa^{-1}(a)\right)=\kappa^{-1}(a) * f_{i h}, \\
\kappa^{-1}\left(a *\left(f_{j i} \circ \kappa^{-1}\right)\right) & =\left(f_{j i} \otimes \mathrm{id}\right)\left(\kappa^{-1} \otimes \kappa^{-1}\right) \Phi(a) \\
& =\left(\mathrm{id} \otimes f_{j i}\right) \Phi\left(\kappa^{-1}(a)\right)=f_{j i} * \kappa^{-1}(a) .
\end{aligned}
$$

Inserting these data into (2.45) and replacing $a$ by $\kappa(a)$ we obtain (2.39). Q.E.D.

Applying $\Phi_{\Gamma}$ to both sides of (2.42) and using (2.2), (2.10), (A.2), and (2.36) we obtain

$$
\Phi_{\Gamma}\left(\eta_{j}\right)=\sum_{i \in I} \kappa\left(R_{i j}\right) \otimes \eta_{i}
$$

Theorem 2.4 gives the complete description of bicovariant bimodules. By virtue of (2.5) and (2.35),

$$
{ }_{\Gamma} \Phi\left(\sum_{i} a_{i} \omega_{i}\right)=\sum_{i, j} \Phi\left(a_{i}\right)\left(\omega_{j} \otimes R_{j i}\right) .
$$

One can prove the following

Theorem 2.5. Let $\left(f_{i j}\right)_{i j \in I}$ be a family of functionals defined on A satisfying relations (2.15) and (2.16) and $\left(R_{i j}\right)_{i j \in I}$ be a family of elements of $\mathscr{A}$ satisfying (2.36), (2.37), and (2.39). We consider the free left module $\Gamma$ over $\mathscr{A}$ generated by $\omega_{i}(i \in I)$ and use formulae (2.29), (2.30), and (2.47) to introduce right multiplication by elements of $\mathscr{A}$ and left and right actions of $G$ on $\Gamma$. Then $\left(\Gamma, \Phi_{\Gamma},{ }_{\Gamma} \Phi\right)$ is a bicovariant bimodule.

Let $\mathscr{A}^{2}$ be the bimodule introduced in Sect. 1. In order to prove Theorems 1.5, 1.6, and 1.8 we have to introduce left and right actions of $G$ on $\mathscr{A}^{2}$. 
Let $q \in \mathscr{A} \otimes \mathscr{A}$ and $(\Phi \otimes \Phi)(q)=\sum_{k} a_{k} \otimes b_{k} \otimes c_{k} \otimes d_{k}, \quad$ where $a_{k}, b_{k}, c_{k}, d_{k} \in \mathscr{A}$ $(k=1,2, \ldots, K)$. We set

$$
\begin{aligned}
& \Phi_{L}(q)=\sum_{k} a_{k} c_{k} \otimes b_{k} \otimes d_{k}, \\
& \Phi_{R}(q)=\sum_{k} a_{k} \otimes c_{k} \otimes b_{k} d_{k} .
\end{aligned}
$$

We claim that $\Phi_{L}(q) \in \mathscr{A} \otimes \mathscr{A}^{2}$ and $\Phi_{R}(q) \in \mathscr{A}^{2} \otimes \mathscr{A}$ for any $q \in \mathscr{A}^{2}$. Indeed, the multiplicativity of $\Phi$ immediately implies that $(\mathrm{id} \otimes m) \Phi_{L}(q)=\Phi(m q)=0$ and $(m \otimes \mathrm{id}) \Phi_{R}(q)=\Phi(m q)=0$. Therefore,

$$
\begin{aligned}
& \Phi_{L}: \mathscr{A}^{2} \rightarrow \mathscr{A} \otimes \mathscr{A}^{2}, \\
& \Phi_{R}: \mathscr{A}^{2} \rightarrow \mathscr{L}^{2} \otimes \mathscr{A}
\end{aligned}
$$

are linear maps. Using (A.1), (A.5), and (A.6) one can check that $\left(\mathscr{A}^{2}, \Phi_{L}, \Phi_{R}\right)$ is a bicovariant bimodule.

Let $r$ and $s$ be bijections introduced by (1.21) and (1.22). Using (A.15) and (A.16) one can easily show that

$$
r\left(\mathscr{A}^{2}\right)=\mathscr{A} \otimes(\text { ker } e)
$$

and $s\left(\mathscr{A}^{2}\right)=($ kere $e) \otimes \mathscr{A}$.

Proposition 2.6. The diagrams

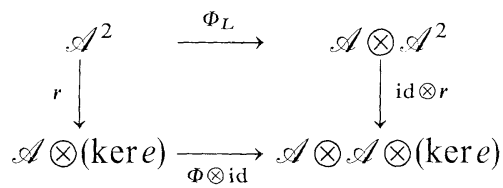

and

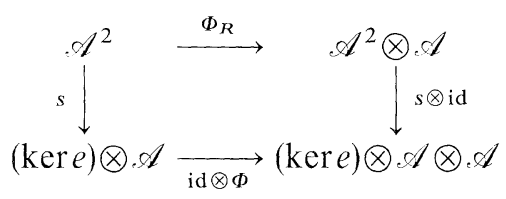

are commutative.

Proof. We claim that

$$
\begin{aligned}
& r=(\mathrm{id} \otimes e \otimes \mathrm{id}) \Phi_{L}, \\
& s=(e \otimes \mathrm{id} \otimes \mathrm{id}) \Phi_{R} .
\end{aligned}
$$

Indeed, if $a, c \in \mathscr{A}$ and

$$
\begin{aligned}
& \Phi(a)=\sum_{k} a_{k} \otimes b_{k}, \\
& \Phi(c)=\sum_{l} c_{l} \otimes d_{l},
\end{aligned}
$$

where $a_{k}, b_{k}, c_{l}, d_{l} \in \mathscr{A}(k=1,2, \ldots, K ; l=1,2, \ldots, L)$, then

$$
\begin{aligned}
& \Phi_{L}(a \otimes \mathrm{c})=\sum_{k, l} a_{k} c_{l} \otimes b_{k} \otimes d_{l}, \\
& \Phi_{R}(a \otimes \mathrm{c})=\sum_{k, l} a_{k} \otimes c_{l} \otimes b_{k} d_{l},
\end{aligned}
$$


and using (A.6) and (A.5) we have

$$
\begin{aligned}
& (\mathrm{id} \otimes e \otimes \mathrm{id}) \Phi_{L}(a \otimes \mathrm{c})=\sum_{k, l} a_{k} e\left(b_{k}\right) c_{l} \otimes d_{l}=\sum_{l} a c_{l} \otimes d_{l}=r(a \otimes c), \\
& (e \otimes \mathrm{id} \otimes \mathrm{id}) \Phi_{R}(a \otimes \mathrm{c})=\sum_{k, l} c_{l} \otimes e\left(a_{k}\right) b_{k} d_{l}=\sum_{l} c_{l} \otimes a d_{l}=s(a \otimes c) .
\end{aligned}
$$

The commutativity of (2.51) follows easily from (2.53) and Condition 2 of Definition $2.1\left[\left(\mathscr{A}^{2}, \Phi_{L}\right)\right.$ is a left-covariant bimodule]. Similarly, the commutativity of (2.52) follows from (2.54) and Condition 2 of Definition $2.2\left[\left(\mathscr{A}^{2}, \Phi_{R}\right)\right.$ is a right-covariant bimodule]. Q.E.D.

Using (A.23) and commutativity of (2.51) and (2.52) one easily obtains

Proposition 2.7. An element of $\mathscr{A}^{2}$ is left- (right-, respectively) invariant if and only if it is of the form $r^{-1}(I \otimes x)\left(s^{-1}(y \otimes I)\right.$, respectively) where $x \in \operatorname{ker} e(y \in \operatorname{ker} e$, respectively).

By virtue of Condition 3 of Definition 2.3 the right shifts applied to leftinvariant elements produce left-invariant elements. Therefore (cf. Proposition 2.7), for any $x \in$ kere there exists $q \in($ ker $e) \otimes \mathscr{A}$ such that

$$
\Phi_{R} r^{-1}(I \otimes x)=\left(r^{-1} \otimes \mathrm{id}\right)(I \otimes q) .
$$

Applying $(e \otimes \mathrm{id} \otimes \mathrm{id})$ to the both sides of this equation and using (2.54), (1.28), and (A.19) we get

$$
\operatorname{ad} x=q .
$$

This way we proved that

$$
\Phi_{R} r^{-1}(I \otimes x)=\left(r^{-1} \otimes \mathrm{id}\right)(I \otimes \mathrm{ad} x)
$$

for any $x \in$ kere.

Now we can prove theorems of Sect. 1 . Let $(\Gamma, d)$ be a first order differential calculus on $G$ and $\mathscr{N}$ be a sub-bimodule of $\mathscr{A}^{2}$ introduced by (1.7). Let us notice that implication $(1.8)\left[(1.16)\right.$, respectively] means that $\Phi_{L}(\mathscr{N}) \subset \mathscr{A} \otimes \mathscr{N}$ $\left[\Phi_{R}(\mathscr{N}) \subset \mathscr{N} \otimes \mathscr{A}\right.$, respectively].

Proof of Theorem 1.5. Let $\mathscr{R}$ be a right ideal of $\mathscr{A}$ such that $\mathscr{R} C$ ker $e$. According to (A.11) and (A.12) $r^{-1}(\mathscr{A} \otimes \mathscr{R})$ is a sub-bimodule of $\mathscr{A}^{2}$. Assume that $\mathscr{N}=r^{-1}(\mathscr{A} \otimes \mathscr{R})$. Then using $(2.51)$ we see that $\Phi_{L}(\mathscr{N})=\left(\mathrm{id} \otimes r^{-1}\right)(\Phi(\mathscr{A}) \otimes \mathscr{R})$ $C\left(\mathrm{id} \otimes r^{-1}\right)(\mathscr{A} \otimes \mathscr{A} \otimes \mathscr{R})=\mathscr{A} \otimes \mathscr{N}$. This shows that the implication (1.8) holds.

Conversely, if (1.8) holds then $\left(\mathscr{N}, \Phi_{L}\right)$ is a left-covariant bimodule and (cf. Theorem 2.1.1 and Proposition 2.7) there exists a family $\left(x_{i}\right)_{i \in I}$ of elements of kere such that $\mathcal{N}$ coincides with the set of all elements of the form

$$
q=\sum_{i \in I} a_{i} r^{-1}\left(I \otimes x_{i}\right)
$$

where $a_{i} \in \mathscr{A}$. Using (1.4) and (A.17) one can easily check that the right-hand side of the above relation equals $r^{-1}\left(\sum_{i} a_{i} \otimes x_{i}\right)$. Denoting by $\mathscr{R}$ the linear span of $x_{i}$ we
obtain $\mathscr{N}=r^{-1}(\mathscr{A} \otimes \mathscr{R})$. 
We have to show that $\mathscr{R}$ is a right ideal in $\mathscr{A}$. Let $x \in \mathscr{R}$ and $a \in \mathscr{A}$. Then $r^{-1}(I \otimes x) \in \mathscr{N}$ and $\left(\mathscr{N}\right.$ is a bimodule) $\left[r^{-1}(I \otimes x)\right](I \otimes a) \in \mathscr{N}$. Therefore [cf. $(\mathrm{A} .12)],(I \otimes x) \Phi(a) \in \mathscr{A} \otimes \mathscr{R}$ and $(e \otimes \mathrm{id})[(I \otimes x) \Phi(a)]=x a \in \mathscr{R} . \quad$ Q.E.D.

Proof of Theorem 1.6. Let $\mathscr{R}$ be a right ideal of $\mathscr{A}$ such that $\mathscr{R} \subset$ kere. According to (A.13) and (A.14) $s^{-1}(\mathscr{R} \otimes \mathscr{A})$ is a sub-bimodule of $\mathscr{A}^{2}$. Assume that $\mathscr{N}=s^{-1}(\mathscr{R} \otimes \mathscr{A})$. Then using $(2.52)$ we see that $\Phi_{R}(\mathscr{N})=\left(s^{-1} \otimes \mathrm{id}\right)(\mathscr{R} \otimes \Phi(\mathscr{A}))$ $C\left(s^{-1} \otimes \mathrm{id}\right)(\mathscr{R} \otimes \mathscr{A} \otimes \mathscr{A})=\mathscr{N} \otimes \mathscr{A}$. This shows that the implication (1.16) holds.

Conversely, if (1.16) holds then $\left(\mathscr{N}, \Phi_{R}\right)$ is a right-covariant bimodule and (cf. Theorem 2.3.1 and Proposition 2.7) there exists a family $\left(y_{i}\right)_{i \in I}$ of elements of kere such that $\mathscr{N}$ coincides with the set of all elements of the form

$$
q=\sum_{i \in I} a_{i} s^{-1}\left(y_{i} \otimes I\right),
$$

where $a_{i} \in \mathscr{A}$. Using (1.4) and (A.18) one can easily check that the right-hand side of the above relation equals $s^{-1}\left(\sum_{i} y_{i} \otimes a_{i}\right)$. Denoting by $\mathscr{R}$ the linear span of $y_{i}$ we obtain $\mathscr{N}=s^{-1}(\mathscr{R} \otimes \mathscr{A})$.

We have to show that $\mathscr{R}$ is a right ideal in $\mathscr{A}$. Let $y \in \mathscr{R}$ and $b \in \mathscr{A}$. Then $s^{-1}(y \otimes I) \in \mathscr{N}$ and $\left(\mathscr{N}\right.$ is a bimodule) $\left[s^{-1}(y \otimes I)\right](I \otimes b) \in \mathscr{N}$. Therefore [cf. (A.14)], $(y \otimes I) \Phi(b) \in \mathscr{R} \otimes \mathscr{A}$ and $(\mathrm{id} \otimes e)[(y \otimes I) \Phi(b)]=y b \in \mathscr{R}$. Q.E.D.

Proof of Theorem 1.8. Let $\mathscr{R}$ be a right ideal of $\mathscr{A}$ such that $\mathscr{R} \subset$ kere and

$$
\mathscr{N}=r^{-1}(\mathscr{A} \otimes \mathscr{R}) \text {. }
$$

Then (cf. proof of Theorem 1.5) $\left(\mathscr{N}, \Phi_{L}\right)$ is a left-covariant bimodule. Assume that $\mathscr{R}$ is ad-invariant. Then formula (2.55) shows (cf. Proposition 2.7) that the $\operatorname{set}_{\text {inv }} \mathscr{N}$ of all left-invariant elements of $\mathscr{N}$ is invariant under right shifts: $\Phi_{R}\left(\right.$ inv $\left._{1} \mathscr{N}\right) C_{\text {inv }} \mathscr{N} \otimes \mathscr{A}$. Now the decomposition (2.11) shows that $\Phi_{R}(\mathscr{N}) \subset \mathscr{N} \otimes \mathscr{A}$. This means that the implication (1.16) holds.

Conversely, assume that (1.16) holds. Then (cf. proof of Theorem 1.6) $\mathscr{N}=S^{-1}\left(\mathscr{R}^{\prime} \otimes \mathscr{A}\right)$, where $\mathscr{R}^{\prime}$ is a right ideal of $\mathscr{A}$ contained in kere. Using (2.56), (A.19), and (A.20) one can easily check that $\mathscr{R}^{\prime}=\mathscr{R}$. So we have

$$
\begin{gathered}
r^{-1}(\mathscr{A} \otimes \mathscr{R})=s^{-1}(\mathscr{R} \otimes \mathscr{A}), \\
s r^{-1}(\mathscr{A} \otimes \mathscr{R})=\mathscr{R} \otimes \mathscr{A}, \\
\operatorname{ad}(\mathscr{R})=s r^{-1}(I \otimes \mathscr{R}) \subset \mathscr{R} \otimes \mathscr{A} .
\end{gathered}
$$

The last inclusion means that $\mathscr{R}$ is ad-invariant. Q.E.D.

In many important cases the bimodules that we deal with are endowed with some additional structure. In these cases considering the concept of left- (right-, respectively) covariance we shall always assume that the additional structure is preserved by the left (right, respectively) action of $G$. For example (see Sect. 3), if $\Gamma$ is a graded algebra then $\mathscr{A} \otimes \Gamma(\Gamma \otimes \mathscr{A}$, respectively) carries natural graded algebra structure and $\Phi_{\Gamma}\left({ }_{\Gamma} \Phi\right.$, respectively) should be a graded algebra homomorphism. In what follows we shall (briefly) discuss $*$-structure on the considered bimodules. For example, if a differential calculus $(\Gamma, d)$ on $G$ is a $*$-calculus then $\Gamma$ is a *-bimodule (cf. Proposition 1.9). 
Let $\left(\Gamma, \Phi_{\Gamma}\right)$ be a left-covariant bimodule over $\mathscr{A}$. Assume that $\Gamma$ is provided with a $*$-structure, i.e. $\Gamma$ is a $*$-bimodule. Then $\mathscr{A} \otimes \Gamma$ carries natural $*$-structure:

$$
(a \otimes \varrho)^{*}=a^{*} \otimes \varrho^{*}
$$

for any $a \in \mathscr{A}$ and $\varrho \in \Gamma$. We say that $\left(\Gamma, \Phi_{\Gamma}\right)$ is a left-covariant $*$-bimodule over $\mathscr{A}$ if

$$
\Phi_{\Gamma}\left(\varrho^{*}\right)=\Phi_{\Gamma}(\varrho)^{*} .
$$

In a similar way one introduces the notion of right-covariant $*$-bimodule. In this case (2.57) and (2.58) should be replaced by

$$
\begin{aligned}
(\varrho \otimes a)^{*} & =\varrho^{*} \otimes a^{*}, \\
{ }_{\Gamma} \Phi\left(\varrho^{*}\right) & ={ }_{\Gamma} \Phi(\varrho)^{*},
\end{aligned}
$$

respectively. We say that $\left(\Gamma, \Phi_{\Gamma},{ }_{\Gamma} \Phi\right)$ is a bicovariant $*$-bimodule if $\left(\Gamma, \Phi_{\Gamma},{ }_{\Gamma} \Phi\right)$ is a bicovariant bimodule and if relations (2.58) and (2.60) hold.

Let $\left(\Gamma, \Phi_{\Gamma}\right)$ be a left-covariant $*$-bimodule over $\mathscr{A}$. Then the set ${ }_{\text {inv }} \Gamma$ of all leftinvariant elements of $\Gamma$ is $*$-invariant: $\omega^{*} \epsilon_{\mathrm{inv}} \Gamma$ for any $\omega \epsilon_{\mathrm{inv}} \Gamma$. Therefore, the basis $\left(\omega_{i}\right)_{i \in I}$ considered in Theorem 2.1 can be chosen in such a way that

$$
\omega_{i}^{*}=\omega_{i}
$$

for all $i \in I$. With this choice the functionals $f_{i j}(i, j \in I)$ introduced by Theorem 2.1 satisfy the following relation:

$$
\overline{f_{i j}(a)}=f_{i j}\left(\kappa(a)^{*}\right)
$$

for any $a \in \mathscr{A}$. Indeed, using (2.13) and (2.14) we have

$$
\begin{aligned}
\sum_{j \in I} \omega_{j}\left(f_{i j} * a^{*}\right)^{*} & =\left(\sum_{j \in I}\left(f_{i j} * a^{*}\right) \omega_{j}\right)^{*}=\left(\omega_{i} a^{*}\right)^{*} \\
& =a \omega_{i}=\sum_{j \in I} \omega_{j}\left(\left(f_{i j} \circ \kappa^{-1}\right) * a\right) .
\end{aligned}
$$

Therefore (cf. Theorem 2.1.2),

$$
\left(f_{i j} * a^{*}\right)^{*}=\left(f_{i j} \circ \kappa^{-1}\right) * a .
$$

Replacing $a$ by $\kappa(a)$ and applying $e$ to both sides we obtain [cf. (A.9)] relation (2.62).

Using (2.61) and (2.13) we have

$$
\left(\sum_{i} a_{i} \omega_{i}\right)^{*}=\sum_{i, j}\left(f_{i j} * a_{i}^{*}\right) \omega_{j} .
$$

Conversely, if $\left(\Gamma, \Phi_{\Gamma}\right)$ is left-covariant bimodule over $\mathscr{A}$ and if for some choice of the basis $\left(\omega_{i}\right)_{i \in I}$ the functionals $f_{i j}(i, j \in I)$ introduced by Theorem 2.1 satisfy relation (2.62), then using the above formula to introduce $*$-operation on $\Gamma$ we obtain a left-covariant $*$-bimodule.

The same remarks apply to right-covariant $*$-bimodules.

If $\left(\Gamma, \Phi_{\Gamma}, \Gamma_{\Gamma} \Phi\right)$ is a bicovariant *-bimodule and if the basis $\left(\omega_{i}\right)_{i \in I}$ considered in Theorem 2.3 satisfies condition (2.61), then the elements $R_{i j} \in \mathscr{A}$ introduced by Theorem 2.4.1 are selfadjoint:

$$
R_{i j}^{*}=R_{i j}
$$

for any $i, j \in I$. 
There exists a natural $*$-operation acting on the bimodule $\mathscr{A}^{2}$ introduced by (1.3). For any $q=\sum a_{k} \otimes b_{k} \in \mathscr{A}^{2}$ we set

$$
q^{*}=\sum b_{k}^{*} \otimes a_{k}^{*} .
$$

One can easily check that $q^{*} \in \mathscr{A}^{2}$ and that $\left(\mathscr{A}^{2}, \Phi_{L}, \Phi_{R}\right)$ is a bicovariant *-bimodule.

Remark. The notation (2.64) may lead to a misunderstanding: $\mathscr{A}^{2} \subset \mathscr{A} \otimes \mathscr{A}$ and the *-operation introduced by (2.64) does not coincide with the hermitian conjugation in the tensor product of two copies of $*$-algebra $\mathscr{A}$. We have to stress that in this section the *-operation applied to elements of $\mathscr{A}^{2}$ is always understood in the sense of (2.64).

We know that the $*$-operation applied to left-invariant elements produce leftinvariant elements. Therefore (cf. Proposition 2.7), for any $x \in$ kere there exists $y \in$ kere such that

$$
r^{-1}(I \otimes x)^{*}=r^{-1}(I \otimes y) .
$$

Applying $e \otimes$ id to both sides and using(A.19) and (A.21) we get $y=\kappa(x)^{*}$. Therefore,

$$
r^{-1}(I \otimes x)^{*}=r^{-1}\left(I \otimes \kappa(x)^{*}\right) .
$$

Now we can prove the last theorem of Sect. 1. Let $(\Gamma, d)$ be a first order differential calculus on $G$ and $\mathscr{N}$ be the sub-bimodule of $\mathscr{A}^{2}$ introduced by (1.7). Let us notice that the implication (1.32) means that $\mathscr{N}^{*} C \mathscr{N}$.

Proof of Theorem 1.10. We know that $\mathscr{N}=r^{-1}(\mathscr{A} \otimes \mathscr{R})$. If $\mathscr{N}^{*} C \mathscr{N}$ then for any $x \in \mathscr{R}, r^{-1}(I \otimes \mathrm{x})^{*} \in \mathscr{N}$ and [cf. (2.65)] $\kappa(x)^{*} \in \mathscr{R}$. Conversely, if $\kappa(x)^{*} \in \mathscr{R}$ for any $x \in \mathscr{R}$ then (2.65) shows that $q^{*} \in \mathscr{N}$ for any left-invariant element $q \in \mathscr{N}$. Remembering that (cf. Theorem 2.1.1) any element $q \in \mathscr{N}$ is of the form $q=\sum a_{i} q_{i}$, where $q_{i} \in \mathscr{N}$ are left-invariant we see that $\mathscr{N}^{*} \subset \mathcal{N}$. Q.E.D.

\section{External Algebra}

Assume for the moment that we are dealing with classical differential geometry, i.e. $\mathscr{A}$ is the algebra of all smooth functions defined on a smooth compact manifold $M$ and $\Gamma$ is the $\mathscr{A}$-bimodule of all first order differential forms on $M$. Then higher order differential forms can be introduced in a purely algebraic way. Denoting by $\Gamma^{\wedge n}$ (where $n=2,3, \ldots$ ) the $\mathscr{A}$-bimodule of all differential $n$-forms we have

$$
\Gamma^{\wedge n}=\Gamma^{\otimes n} / S^{n},
$$

where $\Gamma^{\otimes n}$ is the tensor product (over $\mathscr{A}$ ) of $n$-copies of $\Gamma$ and $S^{n}$ is the kernel of the antisymmetrization operation

$$
A_{n}: \Gamma^{\otimes n} \rightarrow \Gamma^{\otimes n}
$$

introduced by the formula

$$
A_{n}=\sum_{p \in P(n)}(\operatorname{sign} p) \Pi_{p},
$$


where $P(n)$ is the permutation group of $n$ elements and $\Pi$ is the natural action of $P(n)$ on $\Gamma^{\otimes n}$. For example, if $\sigma=\Pi_{t}$, where $t$ is the non-trivial element of $P(2)$, then for any $\varrho, \varrho^{\prime} \in \Gamma$,

$$
\sigma\left(\varrho \otimes_{\mathscr{A}} \varrho^{\prime}\right)=\varrho^{\prime} \otimes_{\mathscr{A}} \varrho .
$$

Trying to repeat Definition (3.1) in the non-commutative setting we meet the following difficulty: there is no natural action of $P(n)$ on $\Gamma^{\otimes n}$. In particular, the formula (3.2) is not self-consistent. Indeed, for any $\varrho, \varrho^{\prime} \in \Gamma, a \in \mathscr{A}$ we have $\varrho \otimes_{\mathscr{A}} a \varrho^{\prime}$ $=\varrho a \otimes_{\mathscr{A}} \varrho^{\prime}$, whereas in general $a \varrho^{\prime} \otimes_{\mathscr{A}} \varrho \neq \varrho^{\prime} \otimes_{\mathscr{A}} \varrho a$.

We shall show, however, that for any bicovariant bimodule $\Gamma$ there exists a natural bimodule automorphism $\sigma$ acting on $\Gamma \otimes_{\mathscr{A}} \Gamma$ replacing the flip automorphism (3.2). This automorphism satisfies the braid equation and defines the natural action of the $n$-element braid group [1] on $\Gamma^{\otimes n}$. As a result we shall obtain the usual external algebra formalism.

As in the previous sections $G=(A, u)$ is a compact matrix pseudogroup and $\mathscr{A}$ is the *-subalgebra of $A$ generated by matrix elements of $u$.

Let $T$ be an algebra and

$$
\begin{aligned}
& \Phi_{T}: T \rightarrow \mathscr{A} \otimes T, \\
& { }_{T} \Phi: T \rightarrow T \otimes \mathscr{A}
\end{aligned}
$$

be linear multiplicative mappings. We say that $\left(T, \Phi_{T},{ }_{T} \Phi\right)$ is a bicovariant algebra if $(e \otimes \mathrm{id}) \Phi_{T}=\mathrm{id}$ and $(\mathrm{id} \otimes e)_{T} \Phi=\mathrm{id}$ and if the following diagrams

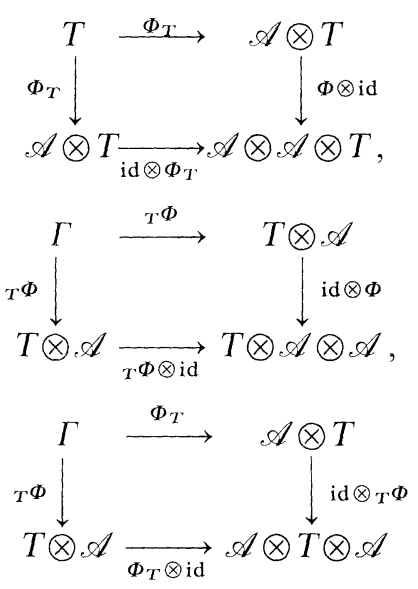

are commutative. Then $\Phi_{T}$ and ${ }_{T} \Phi$ describe left and right actions of $G$ on $T$. For example, $(\mathscr{A}, \Phi, \Phi)$ is a bicovariant algebra.

If $T$ is a graded algebra

$$
T=\sum_{n=0}^{\infty} T^{n}
$$

and the actions of $G$ preserve the grading

$$
\begin{aligned}
& \Phi_{T}\left(T^{n}\right) \subset \mathscr{A} \otimes T^{n}, \\
& { }_{T} \Phi\left(T^{n}\right) \subset T^{n} \otimes \mathscr{A},
\end{aligned}
$$


then we say that $\left(T, \Phi_{T},{ }_{T} \Phi\right)$ is a bicovariant graded algebra. In this case, for any $n=0,1,2, \ldots$ we denote by $\Phi_{T}^{n}\left({ }_{T} \Phi^{n}\right.$, respectively) the restriction of $\Phi_{T}\left({ }_{T} \Phi\right.$, respectively) to $T^{n}$. If $T^{0}=\mathscr{A}$ and $\Phi_{T}^{0}={ }_{T} \Phi^{0}=\Phi$, then for any $n,\left(T^{n}, \Phi_{T}^{n},{ }_{T} \Phi^{n}\right)$ is a bicovariant bimodule over $\mathscr{A}$.

Let $\left(\Gamma, \Phi_{\Gamma},{ }_{\Gamma} \Phi\right)$ be a bicovariant bimodule over $\mathscr{A}$. We say that a bicovariant graded algebra $\left(T, \Phi_{T},{ }_{T} \Phi\right)$ is built over $\left(\Gamma, \Phi_{\Gamma},{ }_{\Gamma} \Phi\right)$ if

1. $T^{0}=\mathscr{A}, \Phi_{T}^{0}={ }_{T} \Phi^{0}=\Phi$.

2. The bicovariant bimodule $\left(T^{1}, \Phi_{T}^{1},{ }_{T} \Phi^{1}\right)$ coincides with $\left(\Gamma, \Phi_{\Gamma},{ }_{\Gamma} \Phi\right)$.

3. $T$ is generated by grade 1 elements, i.e. any element $\tau \in T^{n}(n=2,3, \ldots)$ is of the form $\tau=\sum_{i} \tau_{i}$, where for each $i \tau_{i}$ is a product of $n$ elements of $\Gamma$.

In this section, for any bicovariant bimodule $\left(\Gamma, \Phi_{\Gamma},{ }_{\Gamma} \Phi\right)$ we shall construct two bicovariant graded algebras $\left(\Gamma^{\otimes}, \Phi_{\Gamma}^{\otimes},{ }_{\Gamma} \Phi^{\otimes}\right)$ and $\left(\Gamma^{\wedge}, \Phi_{\Gamma}^{\wedge},{ }_{\Gamma} \Phi^{\wedge}\right)$ built over $\left(\Gamma, \Phi_{\Gamma},{ }_{\Gamma} \Phi\right)$. They are called tensor algebra and external algebra over $\left(\Gamma, \Phi_{\Gamma},{ }_{\Gamma} \Phi\right)$.

We start with the tensor algebra construction. Let

$$
\Gamma^{\otimes n}=\Gamma \otimes_{\mathscr{A}} \Gamma \otimes_{\mathscr{A}} \ldots \otimes_{\mathscr{A}} \Gamma \quad(n \text { factors })
$$

be the $n^{\text {th }}$ tensor power of $\Gamma(n=2,3, \ldots)$. We shall define left and right actions of $G$ on $\Gamma^{\otimes n}$. To this end for any simple element

$$
\tau=\varrho_{1} \otimes_{\mathscr{A}} \varrho_{2} \otimes_{\mathscr{A}} \ldots \otimes_{\mathscr{A}} \varrho_{n}
$$

(where $\varrho_{1}, \varrho_{2}, \ldots, \varrho_{n} \in \Gamma$ ) of $\Gamma^{\otimes n}$ we set

$$
\begin{gathered}
\Phi_{\Gamma}^{\otimes n}(\tau)=\sum_{k} a_{1}^{k_{1}} a_{2}^{k_{2}} \ldots a_{n}^{k_{n}} \otimes\left(\xi_{k_{1}}^{1} \otimes_{\mathscr{A}} \xi_{k_{2}}^{2} \otimes_{\mathscr{A}} \ldots \otimes_{\mathscr{A}} \xi_{k_{n}}^{n}\right), \\
\Gamma^{\otimes n}(\tau)=\sum_{l}\left(\zeta_{l_{1}}^{1} \otimes_{\mathscr{A}} \zeta_{l_{2}}^{2} \otimes_{\mathscr{A}} \ldots \otimes_{\mathscr{A}} \zeta_{l_{n}}^{n}\right) \otimes b_{1}^{l_{1}} b_{2}^{l_{2}} \ldots b_{n}^{l_{n}},
\end{gathered}
$$

where $a_{1}^{k_{1}}, a_{2}^{k_{2}}, \ldots, a_{n}^{k_{n}}, \quad b_{1}^{l_{1}}, b_{2}^{l_{2}}, \ldots, b_{n}^{l_{n}}$ are elements of $\mathscr{A}$ and $\xi_{k_{1}}^{1}, \xi_{k_{2}}^{2}, \ldots, \xi_{k_{n}}^{n}$, $\zeta_{l_{1}}^{1}, \zeta_{l_{2}}^{2}, \ldots, \zeta_{l_{n}}^{n}$ are elements of $\Gamma$ such that

$$
\begin{gathered}
\Phi_{\Gamma}\left(\varrho_{i}\right)=\sum_{k_{\imath}} a_{i}^{k_{\imath}} \otimes \xi_{k_{i}}^{i}, \\
{ }_{\Gamma} \Phi\left(\varrho_{i}\right)=\sum_{l_{l}} \zeta_{l_{\imath}}^{i} \otimes b_{i}^{l_{i}},
\end{gathered}
$$

and the summation [in (3.3) and (3.4)] runs over all possible systems $k=\left(k_{1}, k_{2}, \ldots, k_{n}\right)$ and $l=\left(l_{1}, l_{2}, \ldots, l_{n}\right)$. Taking into account (2.1), (2.2), (2.5), and (2.6) one can easily check that formulae (3.3) and (3.4) introduce well defined linear mappings

$$
\begin{gathered}
\Phi_{\Gamma}^{\otimes n}: \Gamma^{\otimes n} \rightarrow \mathscr{A} \otimes \Gamma^{\otimes n}, \\
\Gamma^{\Phi^{\otimes n}}: \Gamma^{\otimes n} \rightarrow \Gamma^{\otimes n} \otimes \mathscr{A} .
\end{gathered}
$$

Moreover, performing simple but boring computations one can check that $\left(\Gamma^{\otimes n}, \Phi_{\Gamma}^{\otimes n},{ }_{\Gamma} \Phi^{\otimes n}\right)$ is a bicovariant bimodule. For $n=0,1$ we set

$$
\begin{gathered}
\left(\Gamma^{\otimes 0}, \Phi_{\Gamma}^{\otimes 0},{ }_{\Gamma} \Phi^{\otimes 0}\right)=(\mathscr{A}, \Phi, \Phi), \\
\left(\Gamma^{\otimes 1}, \Phi_{\Gamma}^{\otimes 1},{ }_{\Gamma} \Phi^{\otimes 1}\right)=\left(\Gamma, \Phi_{\Gamma}, \Phi\right) .
\end{gathered}
$$


Let

$$
\begin{gathered}
\Gamma^{\otimes}=\sum_{n=0}^{\infty} \Gamma^{\otimes n}, \\
\Phi_{\Gamma}^{\otimes}=\sum_{n=0}^{\infty} \Phi_{\Gamma}^{\otimes n}, \\
\Gamma^{\otimes}=\sum_{n=0}^{\infty} \Gamma^{\oplus} \Phi^{\otimes n} .
\end{gathered}
$$

Clearly, $\Gamma^{\otimes}$ is a graded algebra containing $\mathscr{A}$ as the subalgebra of all elements of grade 0 and $\Gamma$ as the subspace of all elements of grade $1, \Phi_{\Gamma}^{\otimes}$ and ${ }_{\Gamma} \Phi^{\otimes}$ are linear multiplicative maps acting from $\Gamma^{\otimes}$ into $\mathscr{A} \otimes \Gamma^{\otimes}$ and $\Gamma^{\otimes} \otimes \mathscr{A}$, respectively, and $\left(\Gamma^{\otimes}, \Phi_{\Gamma}^{\otimes},{ }_{\Gamma} \Phi^{\otimes}\right)$ is a bicovariant graded algebra built over $\left(\Gamma, \Phi_{\Gamma},{ }_{\Gamma} \Phi\right)$.

To construct the external algebra built over $\left(\Gamma, \Phi_{\Gamma},{ }_{\Gamma} \Phi\right)$ we have to introduce the bimodule automorphism $\sigma$ replacing (3.2).

Proposition 3.1. There exists a unique bimodule homomorphism

$$
\sigma: \Gamma^{\otimes 2} \rightarrow \Gamma^{\otimes 2}
$$

such that

$$
\sigma\left(\omega \otimes_{\mathscr{A}} \eta\right)=\eta \otimes_{\mathscr{A}} \omega
$$

for any left-invariant element $\omega \in \Gamma$ and any right-invariant element $\eta \in \Gamma . \sigma$ is invertible and commutes with the actions of $G$ : the diagrams

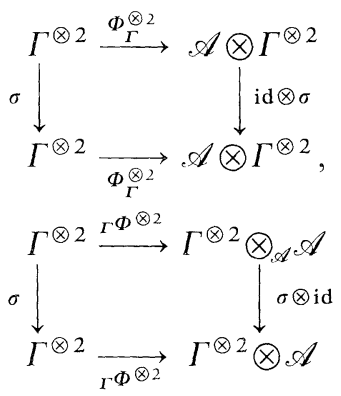

are commutative. Moreover, $\sigma$ satisfies the following braid equation: on $\Gamma^{\otimes 3}$

$$
(\mathrm{id} \otimes \sigma)(\sigma \otimes \mathrm{id})(\mathrm{id} \otimes \sigma)=(\sigma \otimes \mathrm{id})(\mathrm{id} \otimes \sigma)(\sigma \otimes \mathrm{id}) .
$$

Proof. Let $\left(\omega_{i}\right)_{i \in I}\left[\left(\eta_{i}\right)_{i \in I}\right.$, respectively $]$ be the basis in the space of all left- (right-, respectively) invariant elements of $\Gamma$ considered in Theorem 2.4. We shall use

Lemma 3.2. Any element $\tau \in \Gamma^{\otimes 2}$ can be written in the following ways:

$$
\begin{aligned}
\tau & =\sum_{i, j \in I} a_{i j} \omega_{i} \otimes_{\mathscr{A}} \eta_{j}, \\
\tau & =\sum_{i, j \in I} b_{i j} \eta_{j} \otimes_{\mathscr{A}} \omega_{i},
\end{aligned}
$$

where $a_{i j}$ and $b_{i j}(i, j \in I)$ are uniquely determined elements of $\mathscr{A}$. 
Proof of the Lemma. The existence of decompositions (3.9) and (3.10) follows immediately from Theorem 2.1.1 and Theorem 2.3.1.

Let $j \in I$. For any $\varrho \in \Gamma$, we denote by $\theta_{j}(\varrho)$ the element $a_{j} \in \mathscr{A}$ occurring in the decomposition (2.32). Clearly, $\theta_{j}(a \varrho)=a \theta_{j}(\varrho)$ for any $a \in \mathscr{A}$ and $\varrho \in \Gamma$. Due to this fact one can introduce linear mappings id $\otimes \theta_{j}: \Gamma^{\otimes 2} \rightarrow \Gamma$ such that $\left(\mathrm{id} \otimes \theta_{j}\right)\left(\varrho \otimes_{\mathscr{A}} \varrho^{\prime}\right)$ $=\varrho \theta_{j}\left(\varrho^{\prime}\right)$ for any $\varrho, \varrho^{\prime} \in \Gamma$. Applying this mapping to both sides of (3.9) we obtain

$$
\left(\mathrm{id} \otimes \theta_{j}\right)(\tau)=\sum_{i} a_{i j} \omega_{i}
$$

This shows (cf. Theorem 2.1.1) that elements $a_{i j} \in \mathscr{A}(i, j \in I)$ are uniquely determined. Similarly, one can show that elements $b_{i j}$ are uniquely determined. The lemma is proved. Q.E.D.

If a bimodule automorphism $\sigma$ acting on $\Gamma^{\otimes 2}$ satisfies relation (3.5) then

$$
\sigma\left(\sum_{i, j \in I} a_{i j} \omega_{i} \otimes_{\mathscr{A}} \eta_{j}\right)=\sum_{i, j \in I} a_{i j} \eta_{j} \otimes_{\mathscr{A}} \omega_{i}
$$

for any $a_{i j}(i, j \in I)$ belonging to $\mathscr{A}$. On the other hand, using Lemma 3.2 one can easily check that formula (3.11) defines a bijective linear mapping of $\Gamma^{\otimes 2}$ onto itself. We have to show that $\sigma$ is a bimodule homomorphism, i.e. that

$$
\begin{gathered}
\sigma(a \tau)=a \sigma(\tau), \\
\sigma(\tau b)=\sigma(\tau) b
\end{gathered}
$$

for any $a, b \in \mathscr{A}$ and $\tau \in \Gamma^{\otimes 2}$. Relation (3.12) follows immediately from definition (3.11). Taking into account the linearity of $\sigma$ and relation (3.12) we see that it is sufficient to show (3.13) for $\tau=\omega_{i} \otimes_{\mathscr{A}} \eta_{j}(i, j \in I)$. Using (2.13), (2.33) and the associativity of the convolution product we obtain

$$
\begin{aligned}
\sigma\left(\left(\omega_{i} \otimes_{\mathscr{A}} \eta_{j}\right) b\right) & =\sigma\left(\sum_{l, k \in I}\left(f_{i l} * b * f_{j k}\right) \omega_{l} \otimes_{\mathscr{A}} \eta_{k}\right) \\
& =\sum_{l, k \in I}\left(f_{i l} * b * f_{j k}\right) \eta_{k} \otimes_{\mathscr{A}} \omega_{l}=\left(\eta_{j} \otimes_{\mathscr{A}} \omega_{i}\right) b \\
& =\sigma\left(\omega_{i} \otimes_{\mathscr{A}} \eta_{j}\right) b
\end{aligned}
$$

and (3.13) follows.

Assume that $\tau \in \Gamma^{\otimes 2}$ is given by (3.9). Applying $\Phi_{\Gamma}^{\otimes 2}$ to both sides of (3.11) and using (2.46) and (2.10) we have

$$
\begin{aligned}
\Phi_{\Gamma}^{\otimes 2}(\sigma(\tau)) & =\sum_{i j k} \Phi\left(a_{i j}\right)\left[\kappa\left(R_{k j}\right) \otimes\left(\eta_{k} \otimes_{\mathscr{A}} \omega_{i}\right)\right] \\
& =(\mathrm{id} \otimes \sigma)\left(\sum_{i j k} \Phi\left(a_{i j}\right)\left[\kappa\left(R_{k j}\right) \otimes\left(\omega_{i} \otimes_{\mathscr{A}} \eta_{k}\right)\right]\right) \\
& =(\mathrm{id} \otimes \sigma) \Phi_{\Gamma}^{\otimes 2}(\tau) .
\end{aligned}
$$

This shows that the diagram (3.6) is commutative. Similarly, using (2.31) and (2.35) one can prove the commutativity of (3.7).

Let $\omega^{\prime}$ and $\omega^{\prime \prime}$ be left-invariant elements of $\Gamma$. Then $\omega^{\prime} \otimes_{\mathscr{A}} \omega^{\prime \prime}$ is a left-invariant element of $\Gamma^{\otimes 2}$. Using the commutativity of (3.6) we see that $\sigma\left(\omega^{\prime} \otimes_{\mathscr{A}} \omega^{\prime \prime}\right)$ is left- 
invariant. Therefore [cf. also (3.15)],

$$
\sigma\left(\omega^{\prime} \otimes_{\mathscr{A}} \omega^{\prime \prime}\right)=\sum_{r} \omega_{r}^{\prime \prime} \otimes_{\mathscr{A}} \omega_{r}^{\prime},
$$

where $\omega_{r}^{\prime \prime}, \omega_{r}^{\prime}$ are left-invariant elements of $\Gamma$.

Let $\eta$ be a right invariant element of $\Gamma$. Then

$$
\begin{aligned}
(\mathrm{id} \otimes \sigma)(\sigma \otimes \mathrm{id})(\mathrm{id} \otimes \sigma)\left(\omega^{\prime} \otimes_{\mathscr{A}} \omega^{\prime \prime} \otimes_{\mathscr{A}} \eta\right) & =(\mathrm{id} \otimes \sigma)(\sigma \otimes \mathrm{id})\left(\omega^{\prime} \otimes_{\mathscr{A}} \eta \otimes_{\mathscr{A}} \omega^{\prime \prime}\right) \\
& =(\mathrm{id} \otimes \sigma)\left(\eta \otimes_{\mathscr{A}} \omega^{\prime} \otimes_{\mathscr{A}} \omega^{\prime \prime}\right) \\
& =\sum_{r} \eta \otimes_{\mathscr{A}} \omega_{r}^{\prime \prime} \otimes_{\mathscr{A}} \omega_{r}^{\prime} .
\end{aligned}
$$

On the other hand,

$$
\begin{aligned}
(\sigma \otimes \mathrm{id})(\mathrm{id} \otimes \sigma)(\sigma \otimes \mathrm{id})\left(\omega^{\prime} \otimes_{\mathscr{A}} \omega^{\prime \prime} \otimes_{\mathscr{A}} \eta\right) & =(\sigma \otimes \mathrm{id})(\mathrm{id} \otimes \sigma)\left(\sum_{r} \omega_{r}^{\prime \prime} \otimes_{\mathscr{A}} \omega_{r}^{\prime} \otimes_{\mathscr{A}} \eta\right) \\
& =(\sigma \otimes \mathrm{id})\left(\sum_{r} \omega_{r}^{\prime \prime} \otimes_{\mathscr{A}} \eta \otimes_{\mathscr{A}} \omega_{r}^{\prime}\right) \\
& =\sum_{r} \eta \otimes_{\mathscr{A}} \omega_{r}^{\prime \prime} \otimes_{\mathscr{A}} \omega_{r}^{\prime}
\end{aligned}
$$

and formula (3.8) follows. (Using Theorem 2.1.1 and Theorem 2.3.1 one can easily show that any element of $\Gamma^{\otimes 3}$ is a sum of elements of the form $a \omega^{\prime} \otimes_{\mathscr{A}} \omega^{\prime \prime} \otimes_{\mathscr{A}} \eta$, where $a \in \mathscr{A}, \omega^{\prime}$ and $\omega^{\prime \prime}$ are left-invariant and $\eta$ is right-invariant element of $\Gamma$.) Q.E.D.

It turns out that the action of $\sigma$ can be expressed by a formula closely related to Eq. (2.13). To stress this similarity we introduce the convolution product $f * \varrho$, where $f \in \mathscr{A}^{\prime}$ and $\varrho \in \Gamma$. By definition

$$
f * Q=(\mathrm{id} \otimes f)_{\Gamma} \Phi(\varrho) .
$$

Then using the notation introduced in Theorem 2.1 we have

$$
\sigma\left(\omega_{j} \otimes_{\mathscr{A}} \varrho\right)=\sum_{k \in I}\left(f_{j k} * \varrho\right) \otimes_{\mathscr{A}} \omega_{k}
$$

for any $\varrho \in \Gamma$. In particular, setting $\varrho=\omega_{i}$ and using (2.35) we obtain

$$
\sigma\left(\omega_{j} \otimes_{\mathscr{A}} \omega_{i}\right)=\sum_{k l \in I} f_{j k}\left(R_{l i}\right) \omega_{l} \otimes_{\mathscr{A}} \omega_{k}
$$

To prove (3.14) it is sufficient (cf. Theorem 2.3.1) to consider the case $\varrho=a \eta$, where $a \in \mathscr{A}$ and $\eta$ is a right-invariant element of $\Gamma$. Using (2.13) we have

$$
\omega_{j} \otimes_{\mathscr{A}} Q=\omega_{j} \otimes_{\mathscr{A}} a \eta=\omega_{j} a \otimes_{\mathscr{A}} \eta=\sum_{k \in I}\left(f_{j k} * a\right) \omega_{k} \otimes_{\mathscr{A}} \eta .
$$

Therefore,

$$
\sigma\left(\omega_{j} \otimes_{\mathscr{A}} \varrho\right)=\sum_{k \in I}\left(f_{j k} * a\right) \eta \otimes_{\mathscr{A}} \omega_{k} .
$$

On the other hand, using (2.31) we get ${ }_{\Gamma} \Phi(\varrho)=\Phi(a)(\eta \otimes I), f_{j k} * \varrho=\left(f_{j k} * a\right) \eta$ and (3.14) follows.

Let $n$ be a natural number, $n \geqq 2$. We consider the set

$$
\left\{\sigma_{1}, \sigma_{2}, \ldots, \sigma_{n-1}\right\}
$$


of bimodule automorphisms of $\Gamma^{\otimes n}$ introduced by the formula $(k=1,2, \ldots, n-1)$

$$
\sigma_{k}=\mathrm{id} \otimes \ldots \otimes \mathrm{id} \otimes \sigma \otimes \mathrm{id} \otimes \ldots \otimes \mathrm{id},
$$

where the product contains $n-1$ factors and $\sigma$ occurs in the $k^{\text {th }}$ place. It follows immediately from (3.8) that these automorphisms satisfy the basic braid group relations:

$$
\sigma_{k} \sigma_{k+1} \sigma_{k}=\sigma_{k+1} \sigma_{k} \sigma_{k+1}
$$

for $k=1,2, \ldots, n-1$.

Let $P(n)$ be the permutation group of $n$ elements. We consider the set of nearest neighbour transpositions

$$
\left\{t_{1}, t_{2}, \ldots, t_{n-1}\right\} \text {. }
$$

For any $k=1,2, \ldots, n-1$, the permutation $t_{k}$ transposes $k$ with $k+1$ leaving all other elements of $\{1,2, \ldots, n\}$ in their places.

For any $p \in P(n)$ we denote by $I(p)$ the number of inversed pairs in the sequence $(p(1), p(2), \ldots, p(n))$. Then $p$ is equal to a product of $I(p)$ factors belonging to (3.19):

$$
p=t_{k_{1}} t_{k_{2}} \ldots t_{k_{I(p)}} .
$$

Replacing in this product elements of (3.19) by corresponding elements of (3.16) we obtain a bimodule automorphism of $\Gamma^{\otimes n}$. Due to (3.18) this automorphism is independent of the choice of the decomposition (3.20). It will be denoted by $\Pi_{p}$ :

$$
\Pi_{p}=\sigma_{k_{1}} \sigma_{k_{2}} \ldots \sigma_{k_{I(p)}} .
$$

Clearly,

$$
\Pi_{p q}=\Pi_{p} \Pi_{q}
$$

for any $p, q \in P(n)$ such that $I(p q)=I(p)+I(q)$.

The antisymmetrization $A_{n}$ is a bimodule homomorphism acting on $\Gamma^{\otimes n}$ introduced by the formula

$$
A_{n}=\sum \operatorname{sign}(p) \Pi_{p}
$$

where $\operatorname{sign}(p)=(-1)^{I(p)}$ is the sign of permutation $p$ and the summation runs over all $p \in P(n)$. For $n=1$ we set $A_{1}=\mathrm{id}$ (on $\Gamma$ ).

Let $k$ be a natural number smaller than $n$ and

$$
C_{n k}=\left\{q \in P(n): \begin{array}{l}
q(i)<q(j) \text { for any } i, j=1,2, \ldots, n \\
\text { such that either } i<j \leqq k \text { or } k<i<j
\end{array}\right\} .
$$

One can easily check that $C_{n k}$ consists of $\left(\begin{array}{l}n \\ k\end{array}\right)$ elements. Any permutation $p \in P(n)$ admits unique decomposition

$$
p=q p^{\prime} p^{\prime \prime}
$$

where $q \in C_{n k}$ and $p^{\prime}\left(p^{\prime \prime}\right.$, respectively) is a permutation leaving $k$ first ( $n-k$ last) elements in their places. Clearly, $I(p)=I(q)+I\left(p^{\prime}\right)+I\left(p^{\prime \prime}\right)$. Inserting (3.24) into (3.23) 
and using (3.22) we obtain

$$
A_{n}=A_{n k}\left(A_{k} \otimes A_{n-k}\right)
$$

where

$$
A_{n k}=\sum_{q \in C_{n k}} \operatorname{sign}(q) \Pi_{q} .
$$

Now we can introduce the external algebra built over $\left(\Gamma, \Phi_{\Gamma},{ }_{\Gamma} \Phi\right)$. Let

$$
S^{n}=\operatorname{ker} A_{n}
$$

be the sub-bimodule of $\Gamma^{\otimes n}$ consisting of all elements zeroed by $A_{n}$ and

$$
S=\sum_{n=2}^{\infty} S^{n}
$$

By virtue of (3.25) $S$ is an ideal in the algebra $\Gamma^{\otimes}$. Therefore,

$$
\Gamma^{\wedge}=\Gamma^{\otimes} / S=\sum_{n=0}^{\infty} \Gamma^{\wedge n},
$$

where $\Gamma^{\wedge 0}=\mathscr{A}, \Gamma^{\wedge 1}=\Gamma$, and $\Gamma^{\wedge n}=\Gamma^{\otimes n} / S^{n}$ for $n>1$ is a graded algebra. The multiplication in $\Gamma^{\wedge}$ will be denoted by $\wedge$ (this sign is usually omitted if one of the factors is of grade 0 ).

Taking into account the commutativity of (3.6) and (3.7) we see that $S$ is leftand right-invariant. Therefore, $\Gamma^{\wedge}$ carries the natural left and right actions of $G$. Denoting these actions by $\Phi_{\Gamma}^{\wedge}$ and ${ }_{\Gamma} \Phi^{\wedge}$ we obtain a bicovariant graded algebra $\left(\Gamma^{\wedge}, \Phi_{\Gamma}^{\wedge},{ }_{\Gamma} \Phi^{\wedge}\right)$ built over $\left(\Gamma, \Phi_{\Gamma},{ }_{\Gamma} \Phi\right)$. This is the external algebra that we wanted to construct.

The external algebra construction has the following remarkable functorial property:

Theorem 3.3. Let $\left(\widetilde{\Gamma}, \widetilde{\Phi}_{\Gamma},{ }_{\Gamma} \widetilde{\Phi}\right)$ be a bicovariant bimodule over $\mathscr{A},\left(\widetilde{\Gamma}^{\wedge}, \tilde{\Phi}_{\Gamma}^{\wedge},{ }_{\Gamma} \widetilde{\Phi}^{\wedge}\right)$ be the external algebra built over $\left(\widetilde{\Gamma}, \widetilde{\Phi}_{\Gamma},{ }_{\Gamma} \widetilde{\Phi}\right), \Gamma$ be a left-and right-invariant sub-bimodule of $\widetilde{\Gamma}, \Phi_{\Gamma}\left({ }_{\Gamma} \Phi\right.$, respectively) be the restriction of $\widetilde{\Phi}_{\Gamma}\left({ }_{\Gamma} \widetilde{\Phi}\right.$, respectively) to $\Gamma$ and $\left(\Gamma^{\wedge}, \Phi_{\Gamma}^{\wedge},{ }_{\Gamma} \Phi^{\wedge}\right)$ be the external algebra built over $\left(\Gamma, \Phi_{\Gamma},{ }_{\Gamma} \Phi\right)$. Then there exists a grade preserving multiplicative embedding

$$
\Gamma^{\wedge} \subset \tilde{\Gamma}^{\wedge}
$$

coinciding with id on elements of grade 0 and with the inclusion $\Gamma \subset \tilde{\Gamma}$ on elements of grade 1. Moreover, (3.30) intertwines the left and the right actions of $G$ on $\Gamma^{\wedge}$ and $\widetilde{\Gamma}^{\wedge}$, respectively.

Proof. It is obvious that the tensor algebra construction obeys the functorial property described in this theorem. Therefore, $\Gamma^{\otimes} \subset \widetilde{\Gamma}^{\otimes}$.

Let $\sigma, \sigma_{k}, \Pi_{p}, A_{n}, S^{n}$, and $S$ be the objects introduced by (3.11), (3.17), (3.21), (3.23), (3.27), and (3.28) and $\tilde{\sigma}, \tilde{\sigma}_{k}, \widetilde{\Pi}_{p}, \tilde{A}_{n}, \widetilde{S}^{n}$, and $\widetilde{S}$ be the corresponding objects related to the bicovariant bimodule $\left(\widetilde{\Gamma}, \widetilde{\Phi}_{\Gamma},{ }_{\Gamma} \widetilde{\Phi}\right)$. It is sufficient [cf. (3.29)] to show that

$$
\tilde{S} \cap \Gamma^{\otimes}=S .
$$


It follows immediately from (3.11) that $\tilde{\sigma}$ restricted to $\Gamma^{\otimes 2}$ coincides with $\sigma$. Therefore, $\tilde{\sigma}_{k}\left(\tilde{\Pi}_{p}, \tilde{A}_{n}\right.$, respectively) restricted to $\Gamma^{\otimes n}$ coincides with $\sigma_{k}\left(\Pi_{p}, A_{n}\right.$, respectively). This shows that

$$
\tilde{S}^{n} \cap \Gamma^{\otimes n}=\left(\operatorname{ker} \tilde{A}_{n}\right) \cap \Gamma^{\otimes n}=\operatorname{ker} A_{n}=S^{n}
$$

and (3.31) follows. Q.E.D.

We end this section with the following result concerning the $*$-structure.

Theorem 3.4. Let $\left(\Gamma, \Phi_{\Gamma},{ }_{\Gamma} \Phi\right)$ be a bicovariant $*$-bimodule over $\mathscr{A}$ and $\left(\Gamma^{\wedge}, \Phi_{\Gamma}^{\wedge},{ }_{\Gamma} \Phi^{\wedge}\right)$ be the external algebra built over $\left(\Gamma, \Phi_{\Gamma},{ }_{\Gamma} \Phi\right)$. Then $\left(\Gamma^{\wedge}, \Phi_{\Gamma}^{\wedge},{ }_{\Gamma} \Phi^{\wedge}\right)$ becomes a bicovariant graded *-algebra. This means that there exists unique antilinear involution

$$
*: \Gamma^{\wedge} \rightarrow \Gamma^{\wedge}
$$

such that

1. * is grade preserving; on elements of grade 0 it coincides with the hermitian conjugation on $\mathscr{A}$, on elements of grade 1 it equals the original $*$-involution on $\Gamma$. 2. * is antimultiplicative in the graded sense, i.e. for any homogeneous elements $\theta, \theta^{\prime} \in \Gamma^{\wedge}$ of grade $n$ and $n^{\prime}$, respectively, we have

$$
\left(\theta \wedge \theta^{\prime}\right)^{*}=(-1)^{n n^{\prime}} \theta^{\prime *} \wedge \theta^{*} .
$$

3. * is bicovariant, i.e.

$$
\begin{gathered}
\Phi_{\Gamma}^{\wedge}\left(\theta^{*}\right)=\left(\Phi_{\Gamma}^{\wedge}(\theta)\right)^{* \otimes *}, \\
{ }_{\Gamma} \Phi^{\wedge}\left(\theta^{*}\right)=\left({ }_{\Gamma} \Phi^{\wedge}(\theta)\right)^{* \otimes *}
\end{gathered}
$$

for any $\theta \in \Gamma^{\wedge}$.

Proof. At first we introduce the $*$-structure on the tensor algebra. On elements of grade 0 and 1 the $*$-operation is already defined $(\mathscr{A}$ is a $*$-algebra and $\Gamma$ is a *-bimodule). For higher order elements we put

$$
\left(\varrho_{1} \otimes_{\mathscr{A}} \varrho_{2} \otimes_{\mathscr{A}} \ldots \otimes_{\mathscr{A}} \varrho_{n}\right)^{*}=S_{n} \varrho_{n}^{*} \otimes_{\mathscr{A}} \ldots \otimes_{\mathscr{A}} \varrho_{2}^{*} \otimes_{\mathscr{A}} \varrho_{1}^{*},
$$

where $\varrho_{1}, \varrho_{2}, \ldots, \varrho_{n} \in \Gamma$ and $S_{n}=\operatorname{sign} t^{n}=(-1)^{n(n-1) / 2}$ is the sign of the permutation

$$
t^{n}=\left(\begin{array}{c}
1,2, \ldots, n \\
n, n-1, \ldots, 1
\end{array}\right)
$$

One can check that (3.34) defines an antilinear involution acting on $\Gamma^{\otimes}$ and that this involution is antimultiplicative in the graded sense and bicovariant. We have to show that this involution can be pulled down to the level of $\Gamma^{\wedge}=\Gamma^{\otimes} / S$. To this end it is sufficient to show that

$$
\tau^{*} \in S
$$

for any $\tau \in S$.

Let $\omega$ ( $\eta$, respectively) be a left- (right-, respectively) invariant element of $\Gamma$. Then $\omega^{*}\left(\eta^{*}\right.$, respectively) is a left-(right-, respectively) invariant element and using 
(3.34) and (3.5) twice we get

$$
\sigma\left(\left(\eta \otimes_{\mathscr{A}} \omega\right)^{*}\right)=-\sigma\left(\omega^{*} \otimes_{\mathscr{A}} \eta^{*}\right)=-\eta^{*} \otimes_{\mathscr{A}} \omega^{*}=\left(\omega \otimes_{\mathscr{A}} \eta\right)^{*}=\left(\sigma^{-1}\left(\eta \otimes_{\mathscr{A}} \omega\right)\right)^{*} .
$$

This shows (cf. Lemma 3.2) that

$$
\sigma\left(\tau^{*}\right)=\left(\sigma^{-1}(\tau)\right)^{*}
$$

for any $\tau \in \Gamma^{\otimes 2}$.

Let $n \geqq 2, p \in P(n)$ and (3.20) be the decomposition of $p$ into the product of nearest neighbour transpositions containing the minimal number of factors. Then

$$
p^{-1}=t_{k_{I(p)}} \ldots t_{k_{2}} t_{k_{1}}
$$

is the similar decomposition for $p^{-1}$. Therefore, using definition (3.21) and formula (3.37) we obtain (for any $\tau \in \Gamma^{\otimes n}$ ):

$$
\begin{aligned}
\Pi_{p}\left(\tau^{*}\right) & =\sigma_{k_{1}} \sigma_{k_{2}} \ldots \sigma_{k_{I(p)}}\left(\tau^{*}\right)=\left(\sigma_{k_{1}}^{-1} \sigma_{k_{2}}^{-1} \ldots \sigma_{k_{I(p)}}^{-1}(\tau)\right)^{*} \\
& =\left(\left(\sigma_{k_{I(p)}} \ldots \sigma_{k_{2}} \sigma_{k_{1}}\right)^{-1}(\tau)\right)^{*}=\left(\left(\Pi_{p^{-1}}\right)^{-1}(\tau)\right)^{*} .
\end{aligned}
$$

Let $q=t^{n} p$ [where $t^{n}$ is introduced by (3.35)]. Then $q p^{-1}=t^{n}$. One can easily check that $I(q)+I\left(p^{-1}\right)=n(n-1) / 2=I\left(t^{n}\right)$. Therefore [cf. (3.22)], $\Pi_{q} \Pi_{p^{-1}}=\Pi_{t^{n}}$ and $\left(\Pi_{p^{-1}}\right)^{-1}=\left(\Pi_{t^{n}}\right)^{-1} \Pi_{q}$. Taking into account this result we get

$$
\Pi_{p}\left(\tau^{*}\right)=\left(\left(\Pi_{t^{n}}\right)^{-1} \Pi_{q}(\tau)\right)^{*} .
$$

Multiplying both sides by sign $p$ and summing up over $p \in P(n)$ we finally obtain

$$
A_{n}\left(\tau^{*}\right)=s_{n}\left(\left(\Pi_{t^{n}}\right)^{-1} A_{n}(\tau)\right)^{*}
$$

for any $\tau \in \Gamma^{\otimes n}$.

If $A_{n}(\tau)=0$ then $A_{n}\left(\tau^{*}\right)=0$ and (3.36) follows. Q.E.D.

Remark. According to (3.27) the quotient $\Gamma^{\wedge n}=\Gamma^{\otimes n} / S^{n}$ can be identified with the range of the antisymmetrization $A_{n}$. This point of view is used in classical differential geometry where higher order differential forms are identified with skew-symmetric covariant tensor fields. In this picture the external product is expressed by the formula [cf. (3.25)]

$$
\tau \wedge \tau^{\prime}=A_{n k}\left(\tau \otimes \tau^{\prime}\right)
$$

where $\tau \in A_{k} \Gamma^{\otimes k}, \tau^{\prime} \in A_{k^{\prime}} \Gamma^{\otimes k^{\prime}}, n=k+k^{\prime}$, and $A_{n k}$ is given by (3.26). Similarly, using (3.38) we see that the *-operation coincides with

$$
A_{n} \Gamma^{\otimes n} \ni \tau \rightarrow S_{n}\left(\left(\Pi_{t^{n}}\right)^{-1} \tau\right)^{*} \in A_{n} \Gamma^{\otimes n} .
$$

\section{Higher Order Differential Calculus}

In this section we show that the external algebra formalism developed in Sect. 3 is well adapted to our differential calculus.

Theorem 4.1. Let $G=(A, u)$ be a compact matrix pseudogroup, $(\Gamma, d)$ be a bicovariant first order differential calculus on $G, \Phi_{\Gamma}$ and ${ }_{\Gamma} \Phi$ be left and right actions of $G$ on $\Gamma$ introduced in Proposition 1.2 and Proposition 1.3, and $\left(\Gamma^{\wedge}, \Phi_{I}^{\wedge},{ }_{\Gamma} \Phi^{\wedge}\right)$ be the external 
algebra built over $\left(\Gamma, \Phi_{\Gamma},{ }_{\Gamma} \Phi\right)$. Then there exists one and only one linear map

$$
d: \Gamma^{\wedge} \rightarrow \Gamma^{\wedge}
$$

such that

1. $d$ increases the grade by one.

2. On elements of grade $0, d$ coincides with the original derivative (1.1).

3. $d$ is a graded derivative:

$$
d\left(\theta \wedge \theta^{\prime}\right)=d \theta \wedge \theta^{\prime}+(-1)^{k} \theta \wedge d \theta^{\prime}
$$

for any $\theta \in \Gamma^{\wedge k}$ and $\theta^{\prime} \in \Gamma^{\wedge}, k=0,1,2, \ldots$.

4.

$$
d(d \theta)=0
$$

for any $\theta \in \Gamma^{\wedge}$.

5. $d$ is bicovariant:

$$
\begin{gathered}
\Phi_{\Gamma}^{\wedge}(d \theta)=(\mathrm{id} \otimes d) \Phi_{\Gamma}^{\wedge}(\theta), \\
{ }_{\Gamma} \Phi^{\wedge}(d \theta)=(d \otimes \mathrm{id}){ }_{\Gamma} \Phi^{\wedge}(\theta)
\end{gathered}
$$

for any $\theta \in \Gamma^{\wedge}$.

Moreover, if $(\Gamma, d)$ is a *-calculus, then endowing $\Gamma^{\wedge}$ with the induced $*$-structure (cf. Proposition 1.9 and Theorem 3.4) we have

6.

$$
d\left(\theta^{*}\right)=(d \theta)^{*}
$$

for any $\theta \in \Gamma^{\wedge}$.

Proof. We shall use the extended bimodule method described in [8].

Let $\mathscr{A} X$ be the free left $\mathscr{A}$-module with one generator $X$ and

$$
\widetilde{\Gamma}=\mathscr{A} X \oplus \Gamma \text {. }
$$

Any element $\tilde{\xi} \in \tilde{\Gamma}$ is of the form

$$
\tilde{\xi}=c X+\xi,
$$

where $c \in \mathscr{A}$ and $\xi \in \Gamma$ are uniquely determined.

We introduce right multiplication by elements of $\mathscr{A}$ : for any $\tilde{\xi} \in \widetilde{\Gamma}$ of the form (4.5) and any $a \in \mathscr{A}$ we set

$$
\tilde{\xi} a=c a X+(c d a+\xi a) .
$$

One can easily check that the left $\mathscr{A}$-module $\tilde{\Gamma}$ endowed with the right multiplication (4.6) is a bimodule over $\mathscr{A}$. Moreover,

$$
d a=X a-a X
$$

for any $a \in \mathscr{A}$.

We introduce left and right actions of $G$ on $\widetilde{\Gamma}$ : for any $\widetilde{\xi} \in \widetilde{\Gamma}$ of the form (4.5) we set

$$
\begin{aligned}
& \widetilde{\Phi}_{\Gamma}(\tilde{\xi})=\Phi(c)(I \otimes X)+\Phi_{\Gamma}(\xi), \\
& { }_{\Gamma} \tilde{\Phi}(\tilde{\xi})=\Phi(c)(X \otimes I)+{ }_{\Gamma} \Phi(\xi) .
\end{aligned}
$$


Then $\left(\widetilde{\Gamma}, \widetilde{\Phi}_{\Gamma}, \Gamma \widetilde{\Phi}\right)$ is a bicovariant bimodule over $\mathscr{A}, \Gamma \subset \widetilde{\Gamma}$ is an invariant subbimodule and $\widetilde{\Phi}_{\Gamma}\left({ }_{\Gamma} \widetilde{\Phi}\right.$, respectively) restricted to $\Gamma$ coincides with $\Phi_{\Gamma}\left({ }_{\Gamma} \Phi\right.$, respectively). Let $\left(\widetilde{\Gamma}^{\wedge}, \widetilde{\Phi}_{\Gamma}^{\wedge},{ }_{\Gamma} \widetilde{\Phi}^{\wedge}\right)$ be the external algebra built over $\left(\Gamma, \widetilde{\Phi}_{\Gamma},{ }_{\Gamma} \widetilde{\Phi}\right)$. We shall use the inclusion

$$
\Gamma^{\wedge} \subset \tilde{\Gamma}^{\wedge}
$$

described in Theorem 3.3.

It follows immediately from (4.8) and (4.9) that $X$ is a left- and right-invariant element of $\widetilde{\Gamma}$. Therefore [cf. Definition (3.5)],

$$
\tilde{\sigma}\left(X \otimes_{\mathscr{A}} X\right)=X \otimes_{\mathscr{A}} X .
$$

This shows that $A_{2}\left(X \otimes_{\mathscr{A}} X\right)=0, X \otimes_{\mathscr{A}} X \in S^{2}$ and

$$
X \wedge X=0 \text {. }
$$

For any $\theta \in \tilde{\Gamma}^{\wedge}$ we put

$$
d \theta=[X, \theta]_{\mathrm{grad}},
$$

where $[X, \theta]_{\mathrm{grad}}$ is the graded commutator

$$
[X, \theta]_{\mathrm{grad}}=\left\{\begin{array}{l}
X \wedge \theta-\theta \wedge X \\
X \wedge \theta+\theta \wedge X
\end{array} \text { if the grade of } \theta \text { is } \begin{array}{l}
\text { even } \\
\text { odd }
\end{array}\right.
$$

We check that the conditions $1-5$ of the theorem are satisfied. Condition 1 is obvious: $X$ is of grade 1 . Condition 2 follows immediately from (4.7). Condition 3 can be verified by direct computation (see e.g. [2] or [8]). Using definition (4.11) one can easily check that

$$
d(d \theta)=X \wedge X \wedge \theta-\theta \wedge X \wedge X
$$

and Condition 4 follows immediately from (4.10). Taking into account the left- and right-invariance of $X$ we obtain Condition 5.

To end this part of the proof we have to show that $d \theta \in \Gamma^{\wedge}$ for any $\theta \in \Gamma^{\wedge}$. We may assume that

$$
\theta=a_{0} d a_{1} \wedge d a_{2} \wedge \ldots \wedge d a_{n},
$$

where $a_{0}, a_{1}, a_{2}, \ldots, a_{n} \in \mathscr{A}$ (according to Condition 2 of Definition 1.1 any element of $\Gamma^{\wedge}$ is a sum of terms of the above form). Using Conditions 2, 3, and 4 we obtain

$$
d \theta=d a_{0} \wedge d a_{1} \wedge \ldots \wedge d a_{n},
$$

and the inclusion $d\left(\Gamma^{\wedge}\right) \subset \Gamma^{\wedge}$ follows. The above formula proves also the uniqueness of $d$.

Assume now that $(\Gamma, d)$ is a $*$-calculus. To prove Condition 6 we may assume that $\theta$ is of the form (4.12). Then [cf. (3.33)]

$$
\theta^{*}=(-1)^{n(n-1) / 2}\left(d a_{n}\right)^{*} \wedge \ldots \wedge\left(d a_{2}\right)^{*} \wedge\left(d a_{1}\right)^{*} a_{0}^{*},
$$

and using (4.1), (4.2), and (1.36) we get

$$
d\left(\theta^{*}\right)=(-1)^{n(n-1) / 2+n}\left(d a_{n}\right)^{*} \wedge \ldots \wedge\left(d a_{2}\right)^{*} \wedge\left(d a_{1}\right)^{*} \wedge\left(d a_{0}\right)^{*} .
$$


On the other hand, taking into account (4.13) and (3.33) we obtain

$$
(d \theta)^{*}=(-1)^{n(n+1) / 2}\left(d a_{n}\right)^{*} \wedge \ldots \wedge\left(d a_{2}\right)^{*} \wedge\left(d a_{1}\right)^{*} \wedge\left(d a_{0}\right)^{*},
$$

and (4.3) follows. Q.E.D.

\section{Commutators, Jacobi Identity, Cartan-Maurer Formula and All That}

Assume at the beginning that $G$ is a Lie group. Let $\mathscr{A}=C^{\infty}(G)$ and $\mathscr{R}$ be the ideal in $\mathscr{A}$ consisting of all functions vanishing with first derivatives at the neutral element of $G$. Then the Lie algebra of $G$ can be identified with the vector space $T$ of all linear functionals on $\mathscr{A}$ killing $I$ ( $I$ denotes the unity of $\mathscr{A}$ ) and $\mathscr{R}$, endowed with the Lie bracket (commutator)

$$
T \times T \ni\left(\chi, \chi^{\prime}\right) \rightarrow\left[\chi, \chi^{\prime}\right] \in T
$$

given by the formula

$$
\left[\chi, \chi^{\prime}\right]=\chi * \chi^{\prime}-\chi^{\prime} * \chi
$$

It follows immediately from (5.1) that the Lie bracket is antisymmetric:

$$
[\chi, \chi]=0
$$

for any $\chi \in T$. Moreover, remembering that the convolution product is associative one can easily verify the Jacobi identity:

$$
\left[\chi,\left[\chi^{\prime}, \chi^{\prime \prime}\right]\right]=\left[\left[\chi, \chi^{\prime}\right], \chi^{\prime \prime}\right]-\left[\left[\chi, \chi^{\prime \prime}\right], \chi^{\prime}\right]
$$

for any $\chi, \chi^{\prime}, \chi^{\prime \prime} \in T$.

Let $\left(\chi_{i}\right)_{i \in I}$ be a basis in $T$. Then there exist left-invariant first order differential forms $\omega_{\imath}(i \in I)$ on $G$ such that for any $a \in \mathscr{A}$,

$$
d a=\sum_{i \in I}\left(\chi_{i} * a\right) \omega_{i}
$$

Moreover, $\left(\omega_{i}\right)_{i \in I}$ is a basis in the space of all left-invariant first order differential forms on $G$. The external derivatives $d \omega_{i}(i \in I)$ are also left-invariant and may be computed with the use of Cartan-Maurer formula

$$
(\mathrm{id} \otimes d) \boldsymbol{\omega}=-\frac{1}{2}[\boldsymbol{\omega}, \boldsymbol{\omega}],
$$

where

$$
\omega=\sum_{i \in I} \chi_{i} \otimes \omega_{i}
$$

is the canonical $T$-valued first order differential form and

$$
[\boldsymbol{\omega}, \boldsymbol{\omega}]=\sum_{i \in I}\left[\chi_{i}, \chi_{j}\right] \otimes\left(\omega_{i} \wedge()_{j}\right) .
$$

One can easily check that $[\omega, \omega]=2 \omega \wedge \boldsymbol{\omega}$, where

$$
\boldsymbol{\omega} \wedge \boldsymbol{\omega}=\sum_{i j \in I}\left(\chi_{i} * \chi_{j}\right) \otimes\left(\omega_{i} \wedge \omega_{j}\right) .
$$

Therefore,

$$
(\mathrm{id} \otimes d) \omega=-\omega \wedge \boldsymbol{\omega} .
$$


In this section we show that the notions and formulae described above remain valid (after necessary modifications) for any bicovariant differential calculus on a compact matrix pseudogroup.

In the rest of this section we assume that $(\Gamma, d)$ is a bicovariant first order differential calculus on a compact matrix pseudogroup $G$ (although Theorem 5.1 and Theorem 5.2.1 and 2 hold also for left-covariant calculi).

Let $\mathscr{R}$ be the right ideal introduced in Theorem 1.5 and

$$
T=\left\{\chi \in \mathscr{A}^{\prime}: \quad \begin{array}{ll}
\chi(I)=0 & \text { and } \\
& \chi(a)=0 \text { for any } \quad a \in \mathscr{R}
\end{array}\right\} .
$$

To simplify the notation we shall assume that $\operatorname{dim}(\mathscr{A} / \mathscr{R})<\infty$. Then $T$ is finite dimensional.

Theorem 5.1. There exists a unique bilinear form

$$
\Gamma \times T \ni(\varrho, \chi) \rightarrow\langle\varrho, \chi\rangle \in \mathbb{C}
$$

such that

$$
\begin{gathered}
\langle a \varrho, \chi\rangle=e(a)\langle\varrho, \chi\rangle, \\
\langle d a, \chi\rangle=\chi(a)
\end{gathered}
$$

for any $a \in \mathscr{A}, \varrho \in \Gamma$, and $\chi \in T$. Moreover, denoting $b y_{\text {inv }} \Gamma$ the set of all left-invariant elements of $\Gamma$ we have

1. For any $\omega \in_{\mathrm{inv}} \Gamma$,

$$
\left(\begin{array}{c}
\langle\omega, \chi\rangle=0 \\
\text { for any } \quad \chi \in T
\end{array}\right) \Rightarrow(\omega=0) \text {. }
$$

2. For any $\chi \in T$,

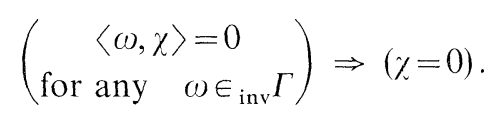

Remark. Statements 1 and 2 mean that $\left\langle_{\text {inv }} \Gamma, T\right\rangle$ is a dual pair of vector spaces. Proof. The uniqueness of (5.8) follows from Condition 2 of Definition 1.1. To prove the existence we shall use the projection

$$
P: \Gamma \rightarrow \Gamma
$$

considered in Lemma 2.2 .

Let $a \in$ kere. If $a_{k}, b_{k}(k=1,2, \ldots, K)$ are elements of $\mathscr{A}$ such that $\Phi(a)=\sum a_{k} \otimes b_{k}$, then $\Phi_{\Gamma}(d a)=\sum a_{k} \otimes d b_{k}$ and formula (2.21) shows that $P d a=\sum \kappa\left(a_{k}\right) d b_{k}$. On the other hand [cf. (1.23) and (2.50)], $\sum \kappa\left(a_{k}\right) \otimes b_{k}=r^{-1}(I \otimes a) \in \mathscr{A}^{2}$, and using the canonical projection $\pi: \mathscr{A}^{2} \rightarrow \Gamma$ introduced in Sect. 1 we obtain

$$
P d a=\pi r^{-1}(I \otimes a)
$$

for any $a \in$ kere. If $P d a=0$ then $r^{-1}(I \otimes a) \in \mathscr{N}$ and $a \in \mathscr{R}$. Conversely, if $a \in \mathscr{R}$, then $r^{-1}(I \otimes a) \in \mathscr{N}$ and $P d a=0$. Therefore, for any $a \in$ kere

$$
(P d a=0) \Leftrightarrow(a \in \mathscr{R}) .
$$


Let $\chi \in T$ and $\varrho \in \Gamma$. Taking into account Condition 2 of Definition 1.1, formula (2.18) and relation $d I=0$ we see that there exists $a \in$ kere such that $P Q=P d a$. We set

$$
\langle\varrho, \chi\rangle=\chi(a) \text {. }
$$

Due to the "only if" part of (5.14) the right-hand side of the above definition is independent of the choice of $a$ [according to (5.7) $\chi$ kills all elements of $\mathscr{R}$ ].

To end the proof of existence we notice that (5.10) is obvious and that (5.9) follows immediately from (2.18).

We claim that

$$
\langle P d a, \chi\rangle=\chi(a)
$$

for any $\chi \in T$ and $a \in \mathscr{A}$. Indeed, if $a \in$ ker $e$ then $P P d a=P d a$ ( $P$ is a projection) and (5.16) is a particular case of (5.15). If $a=\lambda I$ (where $\lambda \in \mathbb{C}$ ) then both sides of (5.16) vanish.

The implication (5.12) is now obvious: $P d a \in_{\mathrm{inv}} \Gamma$ for any $a \in \mathscr{A}$. To prove (5.11) we may assume that $\omega=P d a$, where $a \in$ kere. If $\langle\omega, \chi\rangle=0$ for any $\chi \in T$ then [cf. (5.16) and (5.7)] $a \in \mathscr{R}$ and using the "if" part of (5.14) we get $\omega=0$. Q.E.D.

In the following $\left(\chi_{i}\right)_{i \in I}$ is a basis of $T ;\left(\omega_{i}\right)_{i \in I}$ is the dual basis of ${ }_{\text {inv }} \Gamma$ :

$$
\left\langle\omega_{i}, \chi_{j}\right\rangle=\delta_{i j}
$$

for any $i, j \in I$ and $\left(a_{i}\right)_{i \in I}$ is the family of elements of kere such that

$$
\chi_{i}\left(a_{j}\right)=\delta_{i j}
$$

for any $i, j \in I$. By virtue of (5.16),

$$
\omega_{j}=P d a_{j} .
$$

Clearly,

$$
\omega=\sum_{i \in I}\left\langle\omega, \chi_{i}\right\rangle \omega_{i}
$$

for any $\omega \in_{\mathrm{inv}} \Gamma$. In particular, taking into account (5.16) we get

$$
P d a=\sum_{i \in I} \chi_{i}(a) \omega_{i}
$$

for any $a \in \mathscr{A}$.

Theorem 5.2. 1. For any $a \in \mathscr{A}$,

$$
d a=\sum_{i \in I}\left(\chi_{i} * a\right) \omega_{i}
$$

2. Let $\left(f_{i j}\right)_{i j \in I}$ be the family of linear functionals on $\mathscr{A}$ introduced in Theorem 2.1.3. Then

$$
\chi_{i}(a b)=\sum_{i, j \in I} \chi_{j}(a) f_{j i}(b)+e(a) \chi_{i}(b)
$$

for any $i \in I$ and $a, b \in \mathscr{A}$. In particular,

$$
\chi_{i}\left(a_{j} b\right)=f_{J i}(b)
$$

for any $i, j \in I$ and $b \in \mathscr{A}$. 
3. Let $\left(R_{i j}\right)_{i j \in I}$ be the family of elements of $\mathscr{A}$ introduced in Theorem 2.4.1. Then

$$
R_{i j}=\left(\chi_{i} \otimes \mathrm{id}\right)\left(\operatorname{ad} a_{j}\right)
$$

Proof. Ad1. Let $a_{k}, b_{k}(k=1,2, \ldots, K)$ be elements of $\mathscr{A}$ such that

$$
\Phi(a)=\sum_{k} a_{k} \otimes b_{k} .
$$

Then (cf. Proposition 1.2.4)

$$
\Phi_{\Gamma}(d a)=\sum_{k} a_{k} \otimes d b_{k}
$$

and using (2.19) and (5.18) we get

$$
d a=\sum_{k} a_{k} P d b_{k}=\sum_{i \in I}\left(\sum_{k} a_{k} \chi_{i}\left(b_{k}\right)\right) \omega_{i}=\sum_{i \in I}\left(\chi_{i} * a\right) \omega_{i} .
$$

Ad2. Using (5.19) and (2.13) we obtain

$$
\begin{gathered}
d(a b)=\sum_{i \in I}\left(\chi_{i} * a b\right) \omega_{i}, \\
(d a) b=\sum_{j \in I}\left(\chi_{j} * a\right) \omega_{j} b=\sum_{i j \in I}\left(\chi_{j} * a\right)\left(f_{j i} * b\right) \omega_{i}, \\
a d b=\sum_{i \in I} a\left(\chi_{i} * b\right) \omega_{i} .
\end{gathered}
$$

Inserting these data into (1.2) and using Theorem 2.1.1 we get

$$
\chi_{i} * a b=\sum_{j \in I}\left(\chi_{j} * a\right)\left(f_{j i} * b\right)+a\left(\chi_{i} * b\right),
$$

and using (A.9) we get (5.20).

Ad3. Taking into account Proposition 1.3 .1 and 4 one checks that the canonical mapping (see Sect. 1)

$$
\pi: \mathscr{A}^{2} \rightarrow \Gamma
$$

intertwines the actions $\Phi_{R}$ and ${ }_{\Gamma} \Phi$ of $G$ on $\mathscr{A}^{2}$ and $\Gamma$, respectively. In other words the diagram

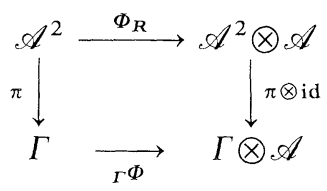

is commutative. Using (5.13), (2.55) and once more (5.13) we have

$$
\begin{aligned}
{ }_{\Gamma} \Phi(P d a) & ={ }_{\Gamma} \Phi \circ \pi\left(r^{-1}(I \otimes \mathrm{a})\right)=(\pi \otimes \mathrm{id})\left(\Phi_{R} r^{-1}(I \otimes \mathrm{a})\right) \\
& =\left(\pi r^{-1} \otimes \mathrm{id}\right)(I \otimes \mathrm{ad} a)=(P d \otimes \mathrm{id})(\operatorname{ad} a) .
\end{aligned}
$$

Therefore [cf. (5.18)],

$$
{ }_{\Gamma} \Phi(P d a)=\sum_{i \in I} \omega_{i} \otimes\left(\chi_{i} \otimes \mathrm{id}\right)(\operatorname{ad} a) .
$$

Inserting $a=a_{j}$, using (5.17) and comparing with (2.35) we obtain (5.21). Q.E.D. 
Now we shall derive some formulae related to the higher order differential calculus. We start with the Cartan-Maurer formula.

Let $a \in \mathscr{A}$. Taking into account (4.2), (5.19), and (4.1) we compute

$$
\begin{aligned}
0 & =d(d a)=d\left(\sum_{i \in I}\left(\chi_{i} * a\right) \omega_{i}\right) \\
& =\sum_{i \in I} d\left(\chi_{i} * a\right) \wedge \omega_{i}+\sum_{i \in I}\left(\chi_{i} * a\right) d \omega_{i} \\
& =\sum_{i \in I}\left(\chi_{j} * \chi_{i} * a\right) \omega_{j} \wedge \omega_{i}+\sum_{i \in I}\left(\chi_{i} * a\right) d \omega_{i} .
\end{aligned}
$$

Applying $P$ to both sides of this relation we get

$$
\sum_{i \in I} \chi_{i}(a) d \omega_{i}=-\sum_{i j \in I}\left(\chi_{j} * \chi_{i}\right)(a) \omega_{j} \wedge \omega_{i}
$$

This shows that

$$
\sum_{i \in I} \chi_{i} \otimes d \omega_{i}=-\sum_{i j \in I}\left(\chi_{j} * \chi_{i}\right) \otimes \omega_{j} \wedge \omega_{i}
$$

and using the short-hand notation (5.5) and (5.6) we obtain the Cartan-Maurer formula

$$
(\mathrm{id} \otimes d) \boldsymbol{\omega}=-\boldsymbol{\omega} \wedge \boldsymbol{\omega} .
$$

Remark. Like in the classical Lie group case the object $\omega \in T \otimes \Gamma$ is independent of the choice of basis $\left(\chi_{i}\right)_{i \in I}$ in $T$.

Inserting in (5.23) $a=a_{k}$ we have

$$
d \omega_{k}=-\sum_{i, j \in I}\left(\chi_{j} * \chi_{i}\right)\left(a_{k}\right) \omega_{j} \wedge \omega_{i}
$$

According to Theorem 2.1.1 any $\tau \in \Gamma^{\otimes 2}$ is of the form

$$
\tau=\sum_{i, j \in I} a_{i j} \omega_{i} \otimes_{\mathscr{A}} \omega_{j},
$$

where $a_{i j} \in \mathscr{A}(i, j \in I)$. Using the same method as in the proof of Lemma 3.2 one can easily show that elements $a_{i j}$ are uniquely determined. Moreover, $\tau$ is left-invariant if and only if $a_{i j}=\lambda_{i j} I$, where $\lambda_{i j} \in \mathbb{C}$ for all $i, j \in I$. It means that ${ }_{\text {inv }} \Gamma^{\otimes 2}$ may be identified with inv $\Gamma \otimes_{\text {inv }} \Gamma$.

Due to this fact there exists a unique bilinear form $\langle\langle\cdot, \cdot\rangle\rangle$ defined on $\left({ }_{\text {inv }} \Gamma^{\otimes 2}\right) \times(T \otimes T)$ such that

$$
\left.\left\langle\left\langle\omega^{\prime} \otimes_{\mathscr{A}} \omega^{\prime \prime}, \chi^{\prime} \otimes \chi^{\prime \prime}\right\rangle\right\rangle=\left\langle\omega^{\prime}, \chi^{\prime}\right\rangle\langle\omega)^{\prime \prime}, \chi^{\prime \prime}\right\rangle
$$

for any $\omega^{\prime}, \omega^{\prime \prime} \in \in_{\text {inv }} \Gamma$ and $\chi^{\prime}, \chi^{\prime \prime} \in T$. This form is non-degenerate, i.e. $\left\langle\left\langle_{\text {inv }} \Gamma^{\otimes 2}, T \otimes T\right\rangle\right.$ is a dual pair of vector spaces.

Let $\sigma: \Gamma^{\otimes 2} \rightarrow \Gamma^{\otimes 2}$ be the bimodule homomorphism introduced in Proposition 3.1. We know that $\sigma$ maps inv $\Gamma^{\otimes 2}$ onto itself. We denote by $\sigma^{t}$ the transpose of $\sigma$. By definition $\sigma^{t}$ is a linear map acting on $T \otimes T$ such that

$$
\left\langle\left\langle\omega^{\prime} \otimes_{\mathscr{A}} \omega^{\prime \prime}, \sigma^{t}\left(\chi^{\prime} \otimes \chi^{\prime \prime}\right)\right\rangle\right\rangle=\left\langle\left\langle\sigma\left(\omega^{\prime} \otimes_{\mathscr{A}} \omega^{\prime \prime}\right), \chi^{\prime} \otimes \chi^{\prime \prime}\right\rangle\right\rangle
$$

for any $\omega^{\prime}, \omega^{\prime \prime} \in{ }_{\mathrm{inv}} \Gamma$ and $\chi^{\prime}, \chi^{\prime \prime} \in T$.

Now we can formulate the analogue of definition (5.1). For any $\chi^{\prime}, \chi^{\prime \prime} \in T$ we set

$$
\left[\chi^{\prime}, \chi^{\prime \prime}\right]=\chi^{\prime} * \chi^{\prime \prime}-\sum_{s} \chi_{s}^{\prime \prime} * \chi_{s}^{\prime}
$$


where $\chi_{s}^{\prime}, \chi_{s}^{\prime \prime}(s=1,2, \ldots, S)$ are elements of $T$ such that

$$
\sigma^{t}\left(\chi^{\prime} \otimes \chi^{\prime \prime}\right)=\sum_{s} \chi_{s}^{\prime \prime} \otimes \chi_{s}^{\prime} .
$$

Theorem 5.3. 1. For any $\chi^{\prime}, \chi^{\prime \prime} \in T$,

$$
\left[\chi^{\prime}, \chi^{\prime \prime}\right] \in T \text {. }
$$
2. If $\chi_{s}^{\prime}, \chi_{s}^{\prime \prime}(s=1,2, \ldots, S)$ are elements of $T$ such that $\sigma^{t}\left(\sum_{s} \chi_{s}^{\prime} \otimes \chi_{s}^{\prime \prime}\right)=\sum_{s} \chi_{s}^{\prime} \otimes \chi_{s}^{\prime \prime}$,
then

$$
\sum_{s}\left[\chi_{s}^{\prime}, \chi_{s}^{\prime \prime}\right]=0
$$

3. For any $\chi, \chi^{\prime}, \chi^{\prime \prime} \in T$,

$$
\left[\chi,\left[\chi^{\prime}, \chi^{\prime \prime}\right]\right]=\left[\left[\chi, \chi^{\prime}\right], \chi^{\prime \prime}\right]-\sum_{s}\left[\left[\chi, \chi_{s}^{\prime \prime}\right], \chi_{s}^{\prime}\right]
$$

where $\chi_{s}^{\prime}, \chi_{s}^{\prime \prime}(s=1,2, \ldots, S)$ are elements of $T$ introduced by (5.26).

Remark. Relation (5.28) expresses the antisymmetry of the commutator [cf. (5.2)], whereas (5.29) plays the role of Jacobi identity [see (5.3)].

Proof. We shall show that

$$
\left[\chi^{\prime}, \chi^{\prime \prime}\right](a)=\left(\chi^{\prime} \otimes \chi^{\prime \prime}\right)(\operatorname{ad} a)
$$

for any $a \in \mathscr{A}$.

To this end we use the extended bimodule language introduced in the proofs of Theorem 3.3 and Theorem 4.1. According to (4.11)

$$
d[P d a]=X \wedge P d a+P d a \wedge X .
$$

On the other hand [cf. (5.23) and (5.18)],

$$
d[P d a]=-\sum_{i . j \in I}\left(\chi_{j} * \chi_{i}\right)(a) \omega_{j} \wedge \omega_{i} .
$$

Combining these two relations we get

$$
X \wedge P d a+P d a \wedge X=-\sum_{i j \in I}\left(\chi_{j} * \chi_{i}\right)(a) \omega_{j} \wedge \omega_{i} .
$$

This means that

$$
X \otimes_{\mathscr{A}} P d a+P d a \otimes_{\mathscr{A}} X \equiv-\sum_{i j \in I}\left(\chi_{j} * \chi_{i}\right)(a) \omega_{j} \otimes_{\mathscr{A}} \omega_{i},
$$

where $\equiv$ denotes the equality modulo $\tilde{S}^{2}=\operatorname{ker} \tilde{A}_{2}=\operatorname{ker}(\mathrm{id}-\tilde{\sigma})$. Therefore,

$$
(\mathrm{id}-\tilde{\sigma})\left(X \otimes_{\mathscr{A}} P d a+P d a \otimes_{\mathscr{A}} X\right)=-(\mathrm{id}-\sigma)\left(\sum_{i j \in I}\left(\chi_{j} * \chi_{i}\right)(a) \omega_{j} \otimes_{\mathscr{A}} \omega_{i}\right) .
$$

At first, we compute the right-hand side of this relation. Let [cf. (3.15)]

$$
\sigma\left(\omega_{i} \otimes_{\mathscr{A}} \omega_{j}\right)=\sum_{k l \in I} \lambda_{i j, k l} \omega_{k} \otimes_{\mathscr{A}} \omega_{l},
$$

where $\lambda_{i j . k l} \in \mathbb{C}(i, j, k, l \in I)$. Then the transpose

$$
\sigma^{t}\left(\chi_{k} \otimes \chi_{l}\right)=\sum_{i j \in I} \lambda_{i j, k l} \chi_{i} \otimes \chi_{j} .
$$


Therefore [cf. (5.25)],

and

$$
\left[\chi_{k}, \chi_{l}\right]=\chi_{k} * \chi_{l}-\sum_{i j \in I} \lambda_{i j, k l} \chi_{i} * \chi_{j}
$$

$$
\begin{gathered}
(\mathrm{id}-\sigma)\left(\sum_{i j \in I}\left(\chi_{j} * \chi_{i}\right)(a) \omega_{j} \otimes_{\mathscr{A}} \omega_{i}\right) \\
=\sum_{k, l \in I}\left(\chi_{k} * \chi_{l}\right)(a) \omega_{k} \bigotimes_{\mathscr{A}} \omega_{l}-\sum_{k, l, i, j}\left(\chi_{i} * \chi_{j}\right)(a) \hat{\lambda}_{i j, k l} \omega_{k} \bigotimes_{\mathscr{A}} \omega_{l} \\
=\sum_{k l \in I}\left[\chi_{k}, \chi_{l}\right](a) \omega_{k} \otimes_{\mathscr{A}} \omega_{l} .
\end{gathered}
$$

To compute the left-hand side of (5.31) we recall that [cf. (4.7) and (5.19)]

$$
X a=a X+\sum_{j \in I}\left(\chi_{j} * a\right) \omega_{j},
$$

and using (3.14) we see that

$$
\tilde{\sigma}\left(X \otimes_{\mathscr{A}} \varrho\right)=\varrho \otimes_{\mathscr{A}} X+\sum_{j \in I}\left(\chi_{j} * \varrho\right) \otimes_{\mathscr{A}} \omega_{j}
$$

for any $\varrho \in \Gamma$. For $\varrho=P d a$ the convolution product on the right-hand side may be computed. By virtue of (5.22)

$$
\chi_{j} * P d a=\left(\mathrm{id} \otimes \chi_{j}\right)_{\Gamma} \Phi(P d a)=\sum_{i \in I}\left(\chi_{i} \otimes \chi_{j}\right)(\operatorname{ad} a) \omega_{i} .
$$

Therefore,

$$
\tilde{\sigma}\left(X \otimes_{\mathscr{A}} P d a\right)=P d a \otimes_{\mathscr{A}} X+\sum_{i j \in I}\left(\chi_{i} \otimes \chi_{j}\right)(\operatorname{ad} a) \omega_{i} \otimes_{\mathscr{A}} \omega_{j} .
$$

Remembering that $P d a$ is left- and $X$ is right-invariant and using (3.5) we get

$$
\tilde{\sigma}\left(P d a \otimes_{\mathscr{A}} X\right)=X \otimes_{\mathscr{A}} P d a .
$$

Combining the last two equations we have

$$
(\mathrm{id}-\tilde{\sigma})\left(X \otimes_{\mathscr{A}} P d a+P d a \otimes_{\mathscr{A}} X\right)=-\sum_{i j \in I}\left(\chi_{i} \otimes \chi_{j}\right)(\operatorname{ad} a) \omega_{i} \otimes_{\mathscr{A}} \omega_{j} .
$$

Inserting this expression and (5.32) into (5.31) we obtain

$$
\left[\chi_{k}, \chi_{l}\right](a)=\left(\chi_{k} \otimes \chi_{l}\right)(\operatorname{ad} a)
$$

and (5.30) follows.

Now the proof of Statement 1 is simple. If $a \in \mathscr{R}$ then according to Theorem 1.8, ad $a \in \mathscr{R} \otimes \mathscr{A}$. If $a=I$ then ad $a=I \otimes I$. In both cases $(5.30)$ shows that $\left[\chi^{\prime}, \chi^{\prime \prime}\right](a)=0$ and (5.27) follows.

Statement 2 is obvious.

To prove Statement 3 we use the commutativity of (1.30). For any $\chi, \chi^{\prime}, \chi^{\prime \prime} \in T$ we have [cf. (5.30)],

$$
\begin{aligned}
{\left[\left[\chi, \chi^{\prime}\right], \chi^{\prime \prime}\right](a) } & =\left(\left[\chi, \chi^{\prime}\right] \otimes \chi^{\prime \prime}\right)(\operatorname{ad} a) \\
& =\left(\chi \otimes \chi^{\prime} \otimes \chi^{\prime \prime}\right)(\operatorname{ad} \otimes \mathrm{id})(\operatorname{ad} a) \\
& =\left(\chi \otimes \chi^{\prime} \otimes \chi^{\prime \prime}\right)(\operatorname{id} \otimes \Phi)(\operatorname{ad} a) \\
& =\left(\chi \otimes \chi^{\prime} * \chi^{\prime \prime}\right)(\operatorname{ad} a) .
\end{aligned}
$$


Using this relation we compute [cf. (5.25)]

$$
\begin{aligned}
{\left[\chi,\left[\chi^{\prime}, \chi^{\prime \prime}\right]\right](a) } & =\left(\chi \otimes\left[\chi^{\prime}, \chi^{\prime \prime}\right]\right)(\operatorname{ad} a) \\
& =\left(\chi \otimes \chi^{\prime} * \chi^{\prime \prime}\right)(\operatorname{ad} a)-\sum_{s}\left(\chi \otimes \chi_{s}^{\prime \prime} * \chi_{s}^{\prime}\right)(\operatorname{ad} a) \\
& =\left[\left[\chi, \chi^{\prime}\right], \chi^{\prime \prime}\right](a)-\sum_{s}\left[\left[\chi, \chi_{s}^{\prime \prime}\right], \chi_{s}^{\prime}\right](a),
\end{aligned}
$$

and (5.29) follows. Q.E.D.

Besides the antisymmetry and the Jacobi identity the commutator obeys an additional relation (that become trivial in the classical differential calculus). To formulate this relation we use the linear mapping

$$
C: T \otimes T \rightarrow T,
$$

such that

$$
C\left(\chi \otimes \chi^{\prime}\right)=\left[\chi, \chi^{\prime}\right]
$$

for any $\chi, \chi^{\prime} \in T$. With this notation we have

\section{Theorem 5.4.}

$$
\begin{aligned}
& \sigma^{t}(\mathrm{id} \otimes C)+\sigma^{t}(C \otimes \mathrm{id})\left(\mathrm{id} \otimes \sigma^{t}\right) \\
& \quad=(C \otimes \mathrm{id})\left(\mathrm{id} \otimes \sigma^{t}\right)\left(\sigma^{t} \otimes \mathrm{id}\right)+(\mathrm{id} \otimes C)\left(\sigma^{t} \otimes \mathrm{id}\right) .
\end{aligned}
$$

Proof. Both sides of (5.36) are mappings from $T \otimes T \otimes T$ into $T \otimes T$. Combining (5.33), (5.34), and (5.16) we get

$$
\begin{aligned}
\tilde{\sigma}\left(X \otimes_{\mathscr{A}} P d a\right) & =P d a \otimes_{\mathscr{A}} X+\sum_{i j \in I}\left[\chi_{i}, \chi_{j}\right](a) \omega_{i} \otimes_{\mathscr{A}} \omega_{j} \\
& =P d a \otimes_{\mathscr{A}} X+\sum_{i j \in I}\left\langle P d a,\left[\chi_{i}, \chi_{j}\right]\right\rangle \omega_{i} \otimes_{\mathscr{A}} \omega_{j} .
\end{aligned}
$$

Therefore, for any $\omega \in_{\mathrm{inv}} \Gamma$,

$$
\tilde{\sigma}\left(X \otimes_{\mathscr{A}} \omega\right)=\omega \otimes_{\mathscr{A}} X+\sum_{i, j \in I}\left\langle\omega,\left[\chi_{i}, \chi_{j}\right]\right\rangle \omega_{i} \otimes_{\mathscr{A}} \omega_{j}=\omega \otimes_{\mathscr{A}} X+C^{t} \omega,
$$

where $C^{t}:_{\text {inv }} \Gamma \rightarrow_{\text {inv }} \Gamma^{\otimes 2}$ denotes the transpose of (5.35).

According to Proposition $3.1 \tilde{\sigma}$ satisfies the braid equation:

$$
(\mathrm{id} \otimes \tilde{\sigma})(\tilde{\sigma} \otimes \mathrm{id})(\mathrm{id} \otimes \tilde{\sigma})=(\tilde{\sigma} \otimes \mathrm{id})(\mathrm{id} \otimes \tilde{\sigma})(\tilde{\sigma} \otimes \mathrm{id}) .
$$

Using (5.37) we are able to compute the action of both sides of (5.38) on $X \otimes_{\mathscr{A}} \tau$, where $\tau \in \in_{\text {inv }} \Gamma^{\otimes 2}$. We obtain

$$
\begin{aligned}
& \sigma \tau \otimes_{\mathscr{A}} X+\left(\mathrm{id} \otimes C^{t}\right) \sigma \tau+(\mathrm{id} \otimes \sigma)\left(C^{t} \otimes \mathrm{id}\right) \sigma \tau \\
& \quad=(\sigma \otimes \mathrm{id})(\mathrm{id} \otimes \sigma)\left(C^{t} \otimes \mathrm{id}\right) \tau+\sigma \tau \otimes \otimes_{\mathscr{A}} X+(\sigma \otimes \mathrm{id})\left(\mathrm{id} \otimes C^{t}\right) \tau .
\end{aligned}
$$

Therefore,

$\left(\mathrm{id} \otimes C^{t}\right) \sigma+(\mathrm{id} \otimes \sigma)\left(C^{t} \otimes \mathrm{id}\right) \sigma=(\sigma \otimes \mathrm{id})(\mathrm{id} \otimes \sigma)\left(C^{t} \otimes \mathrm{id}\right)+(\sigma \otimes \mathrm{id})\left(\mathrm{id} \otimes C^{t}\right)$,

and passing to the transpose mappings we obtain (5.36). Q.E.D. 
Remark. According to (3.5) $\sigma\left(X \otimes_{\mathscr{A}} X\right)=X \otimes_{\mathscr{A}} X$ and for any $\omega \in_{\mathrm{inv}} \Gamma, \sigma\left(\omega \otimes_{\mathscr{A}} X\right)$ $=X \otimes_{\mathscr{A}} \omega$. Using (5.37) one is able to compute the action of both sides of (5.38) on $X \otimes_{\mathscr{A}} X \otimes_{\mathscr{A}} \omega$ where $\omega \in_{\text {inv }} \Gamma$. One gets

$$
\left(C^{t} \otimes \mathrm{id}\right) C^{t} \omega=\left(\mathrm{id} \otimes C^{t}\right) C^{t} \omega+(\mathrm{id} \otimes \sigma)\left(C^{t} \otimes \mathrm{id}\right) C^{t} \omega,
$$

and passing to the transpose mappings one has

$$
C(C \otimes \mathrm{id})=C(\mathrm{id} \otimes C)+C(C \otimes \mathrm{id})\left(\mathrm{id} \otimes \sigma^{t}\right) .
$$

The reader will easily discover that this relation coincides with (5.29). This shows that there exists a logical link between the braid equation and Jacobi identity.

\section{A. Appendix}

Throughout the paper we referred to many formulae obtained in [10]. For the reader's convenience we collect them here. The following list contains also a number of formulae that (although not explicitly contained in [10]) are closely related to results of [10] and can be easily verified.

We use the following notation: $\Phi$ and $\kappa$ are the comultiplication and coinverse associated with a compact matrix pseudogroup $G=(A, u), \mathscr{A}$ is a $*$-algebra generated by matrix elements of $u, e$ is a $*$-character of $\mathscr{A}$ such that $e\left(u_{k l}\right)=\delta_{k l}$, $m: \mathscr{A} \otimes \mathscr{A} \rightarrow \mathscr{A}$ is the multiplication map $[m(a \otimes b)=a b$ for any $a, b \in \mathscr{A}], \sigma_{A}$ is the flip automorphism of $\mathscr{A} \otimes \mathscr{A}\left[\sigma_{A}(a \otimes b)=b \otimes a\right.$ for any $\left.a, b \in \mathscr{A}\right]$, and $r$ and $s$ are linear bijections acting on $\mathscr{A} \otimes \mathscr{A}$ introduced by (1.21) and (1.22). In the formulae below $a, b \in \mathscr{A}, q \in \mathscr{A} \otimes \mathscr{A}, f, f^{\prime}$ are linear functionals defined on $\mathscr{A}$, and * denotes convolution product. We start with

Standard properties of comultiplication and coinverse:

$$
\begin{gathered}
(\Phi \otimes \mathrm{id}) \Phi=(\mathrm{id} \otimes \Phi) \Phi, \\
\Phi \circ \kappa=\sigma_{A}(\kappa \otimes \kappa) \Phi, \\
m(\kappa \otimes \mathrm{id}) \Phi(a)=e(a) I, \\
m(\mathrm{id} \otimes \kappa) \Phi(a)=e(a) I .
\end{gathered}
$$

Properties of $e$ :

$$
\begin{gathered}
(e \otimes \mathrm{id}) \Phi(a)=a, \\
(\mathrm{id} \otimes e) \Phi(a)=a, \\
e \odot \kappa=e, \\
e * a=a * e=a, \\
f * e=e * f=f, \\
e(f * a)=e(a * f)=f(a) .
\end{gathered}
$$

Cyclicity of the convolution product:

$$
f\left(f^{\prime} * a\right)=\left(f * f^{\prime}\right)(a)=f^{\prime}(a * f) .
$$


Properties of bijections $r$ and $s$. These formulae follow immediately from definitions of $r$ and $s$ :

$$
\begin{gathered}
r((a \otimes I) q)=(a \otimes I) r(q), \\
r(q(I \otimes b))=r(q) \Phi(b), \\
s((a \otimes I) q)=(I \otimes a) s(q), \\
s(q(I \otimes b))=s(q) \Phi(b) .
\end{gathered}
$$

Properties of $r^{-1}$ and $s^{-1}$. These formulae follow immediately from (1.23) and the corresponding formulae for $s^{-1}$ (see (4.35) of [10]):

$$
\begin{gathered}
m r^{-1}(q)=(\mathrm{id} \otimes e)(q), \\
m s^{-1}(q)=(e \otimes \mathrm{id})(q), \\
r^{-1}((a \otimes I) q)=(a \otimes I) r^{-1}(q), \\
s^{-1}((I \otimes a) q)=(a \otimes I) s^{-1}(q), \\
(e \otimes \mathrm{id}) r^{-1}(a \otimes b)=e(a) b, \\
(e \otimes \mathrm{id}) s^{-1}(a \otimes b)=a e(b), \\
(\mathrm{id} \otimes e) r^{-1}(a \otimes b)=a \kappa(b) .
\end{gathered}
$$

We end with the following formula

$$
\sum_{k} \Phi\left(\kappa\left(a_{k}\right)\right)\left(b_{k} \otimes I\right)=I \otimes \kappa(a),
$$

where $a_{k}, b_{k}$ are elements of $\mathscr{A}$ such that

$$
\Phi(a)=\sum_{k} a_{k} \otimes b_{k} .
$$

It follows from (A.2), (A.1), and (A.3). Using (A.5) and (A.6) one can easily verify that

$$
(\Phi(a)=I \otimes a) \Leftrightarrow(\Phi(a)=a \otimes I) \Leftrightarrow(a=e(a) I) .
$$

\section{References}

1. Birman, J.: Braids, links, and mapping class groups. Ann. Math. Stud. 82 (1974)

2. Connes, A.: Non-commutative differential geometry. Institut des Hautes Etudes Scientifiques. Extrait des Publications Mathématiques No. 62 (1986)

3. Drinfeld, V.G.: Quantum groups. Proceedings of the International Congress of Mathematicians. Berkeley, CA, USA (1986), pp. 793-820

4. Jimbo, M.: A q-analogue of $U(g l(N+1))$, Hecke algebra and Yang-Baxter equation. Lett. Math. Phys. 11, 247-252 (1986)

5. Koornwinder, T.H.: In preparation

6. Rosso, M.: Comparaison des groupes quantiques de Drinfeld et de Woronowicz. C.R. Acad. Sci. Paris 304, 323-326 (1987)

7. Woronowicz, S.L.: Pseudospaces, pseudogroups and Pontriagin duality. Proceedings of the International Conference on Mathematics and Physics, Lausanne 1979. Lecture Notes in Physics, Vol. 116. Berlin, Heidelberg, New York: Springer 1980 
8. Woronowicz, S.L.: Twisted $S U(2)$ group. An example of a noncommutative differential calculus. Publ. Res. Inst. Math. Sci., Kyoto University 23, 117-181 (1987)

9. Woronowicz, S.L.: Group structure on noncommutative spaces. Fields and Geometry 1986. Proceedings of the XXIInd Winter School and Workshop of Theoretical Physics, Karpacz, Poland, pp. 478-496. Singapore: World Scientific

10. Woronowicz, S.L.: Compact matrix pseudogroups. Commun. Math. Phys. 111, 613-665 (1987)

Communicated by A. Connes

Received July 26, 1988 